\title{
THE MOBILE EXPERIENCE AT RYERSON
}

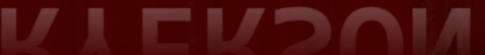




\section{Presentation Outline}

- Humble Beginnings

- Mobile Survey 2008

- What we Learned

- What we Created

- Ryerson mobile

- Mobile Survey 2009

- Library mobile now

- Statistics

- Upcoming Services

- What we might want to do 


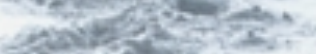

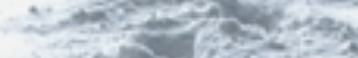

ther $x^{2}-19=$

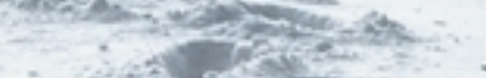
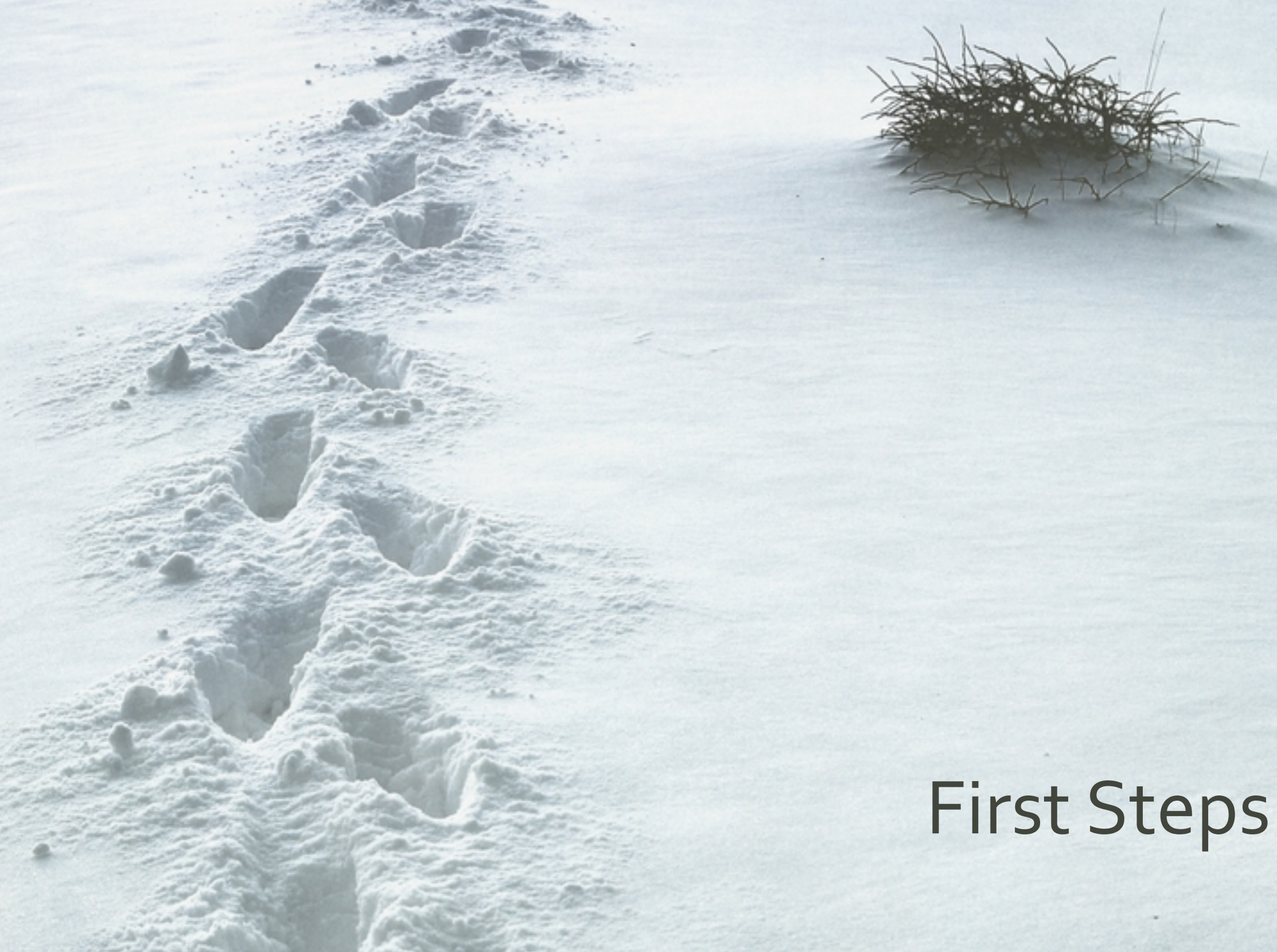

First Steps 


\section{Our humble beginnings...}

Ryerson Library

\section{Mobile Pages}

\section{Hours}

Check for Available Laptops

Library News

Contact Us

Research Workshops

Home | 416-979-5055

Ryerson Library Mobile (circa. Fall 2008)
Send SMS Message

Title: The ghost map : the story of London's most terrifyin!

Call \#: RC133.G6 J64 2006

Floor

Location: 10 th

Select Service

Provider:

Rogers $\quad$

Cell Phone \#: $\quad$ eg. 4161231234

NOTE: carrier charges may apply

Send Message Go Back

SMS from the catalogue (circa. May 2007)

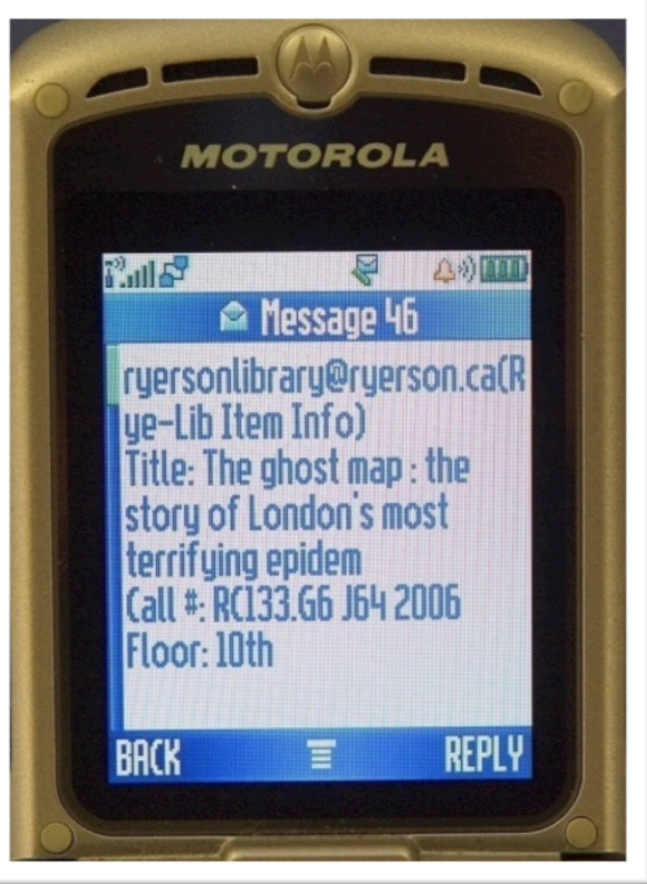




\section{Mobile Survey - Nov. 2008}
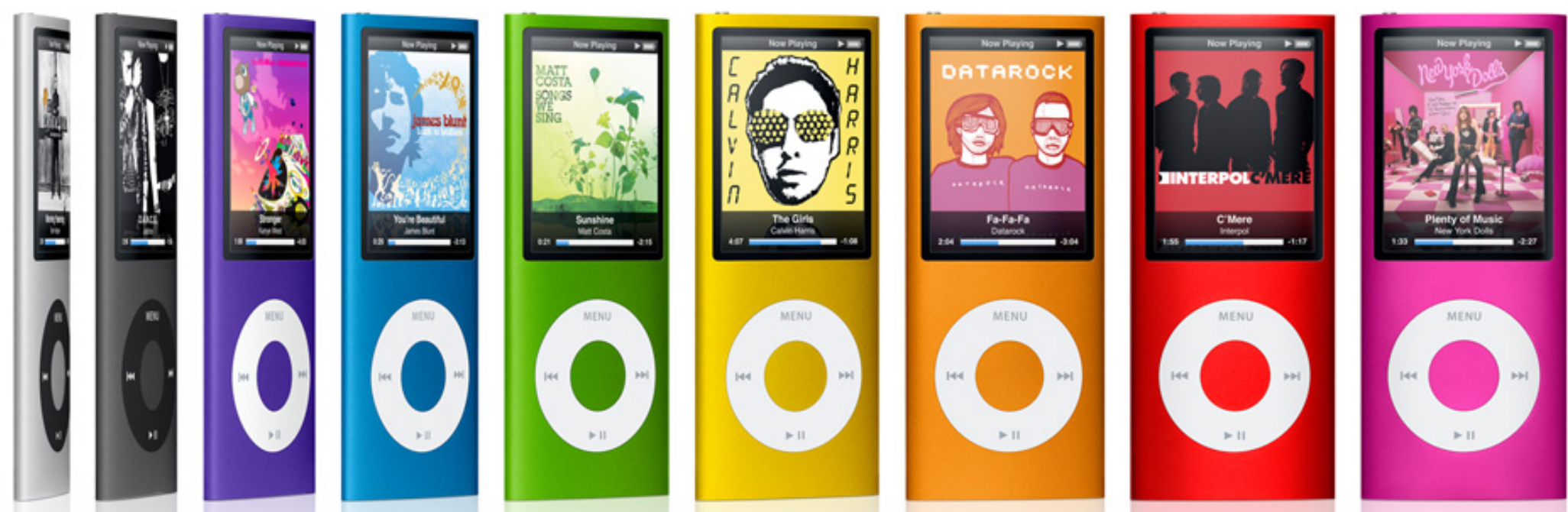

811 responses, primarily undergraduate 


\section{Cell Phone Ownership}

November 2008

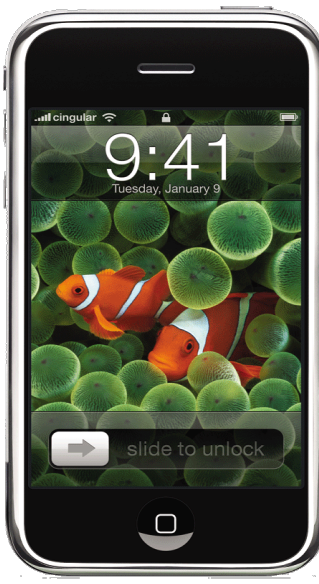

No Phone

$$
\text { 3\% }
$$

Smart Phone $21 \%$

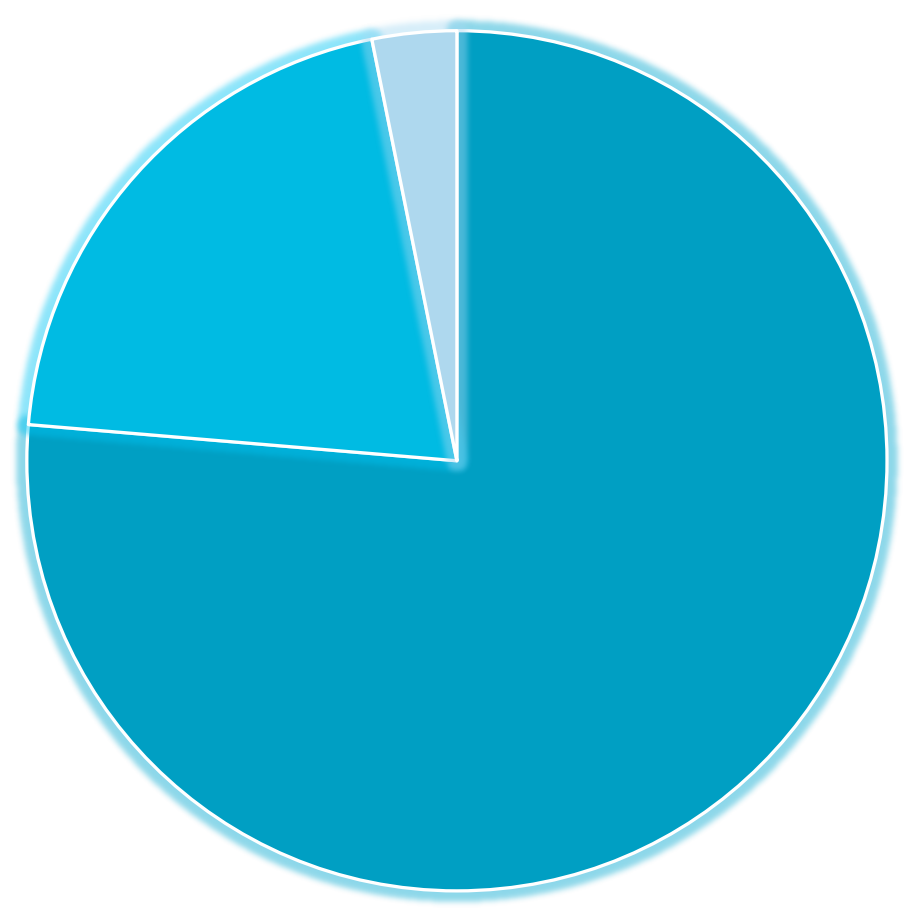

Cell Phone $76 \%$ 


\section{Top 4 Uses}

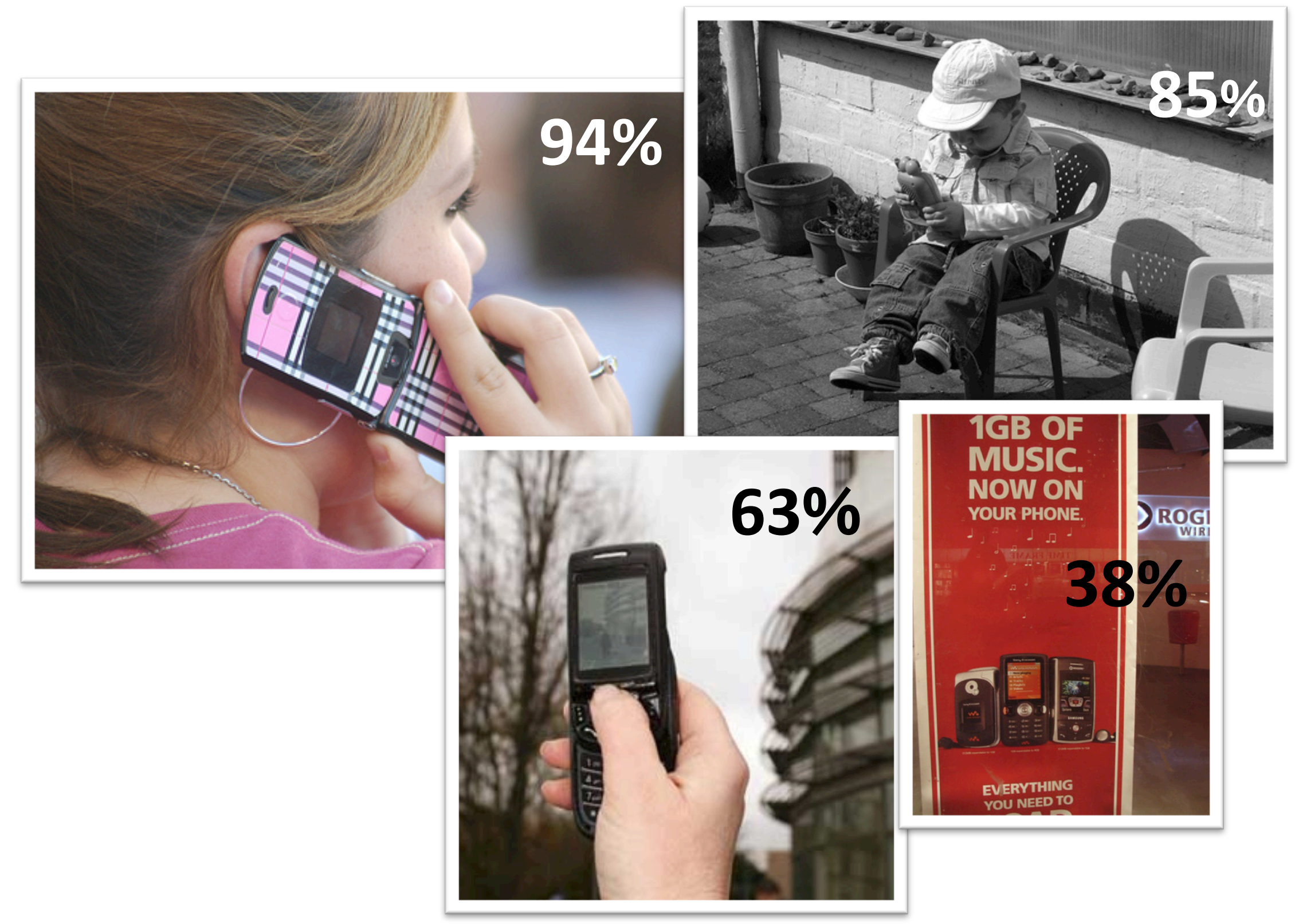




\section{Top Internet Uses}

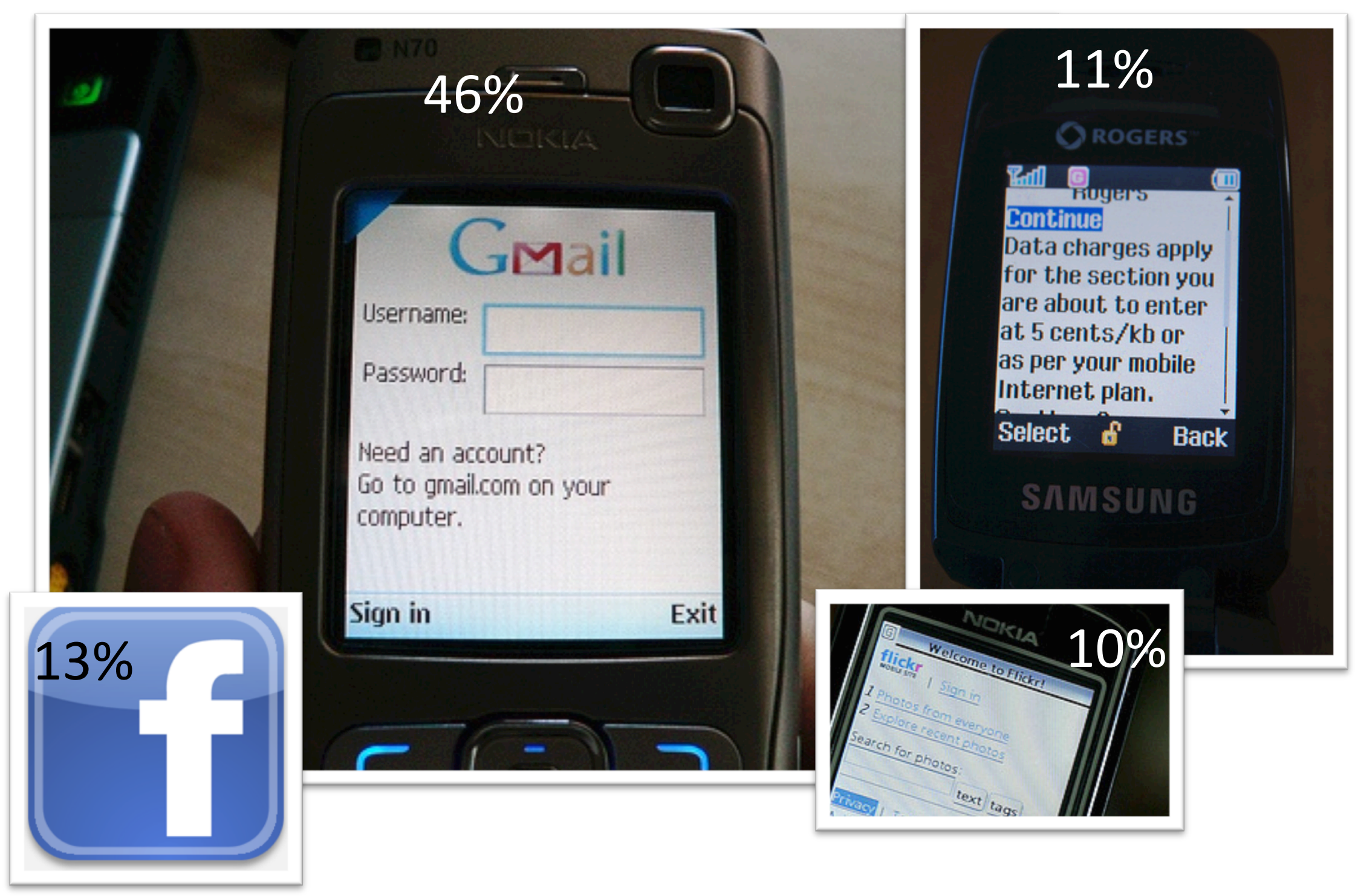




\section{What will your next phone be?}

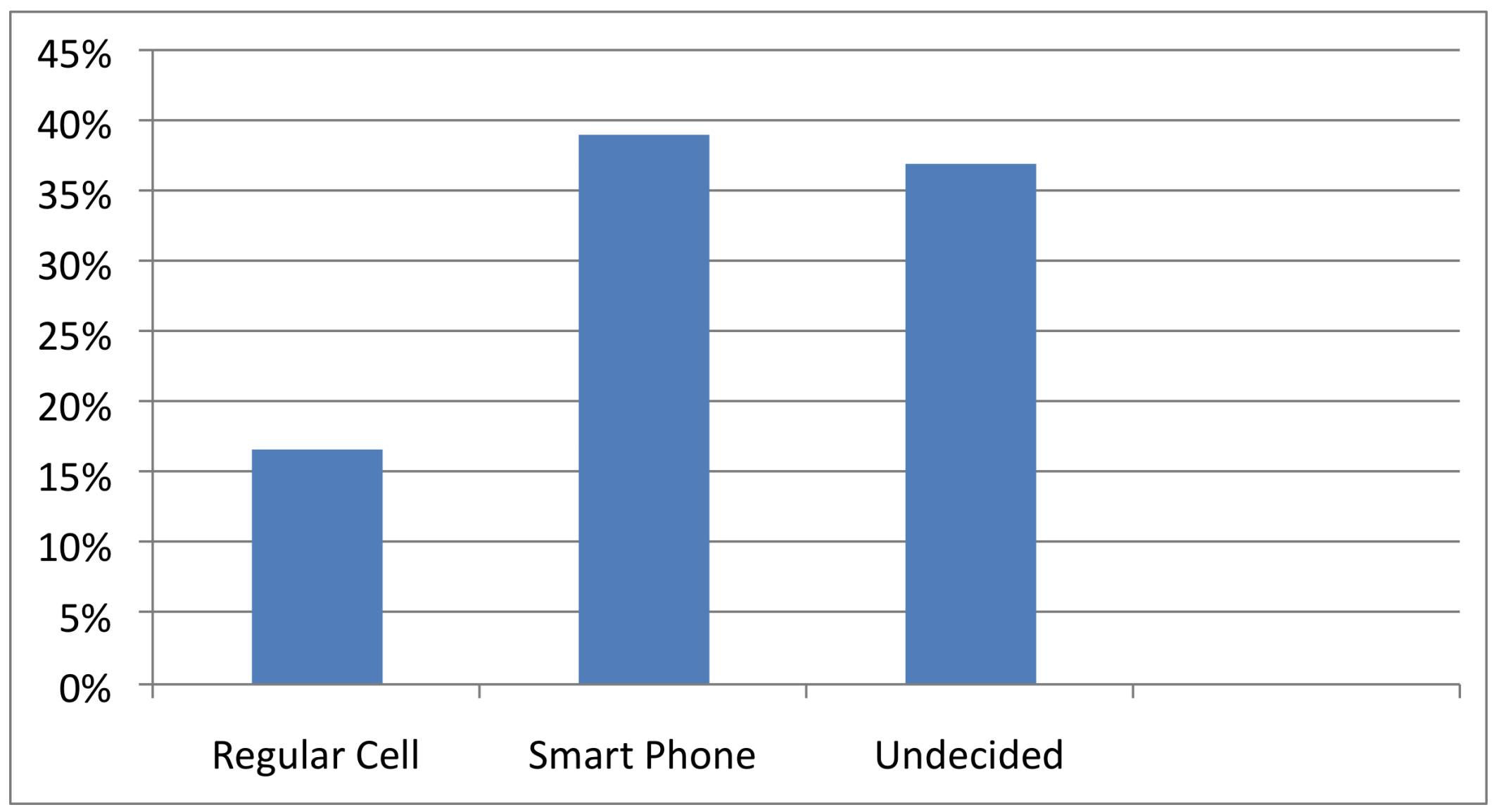




\section{Mobile Survey Novemberzoo8}

\section{What Library Services would you like to access on your mobile devices?}

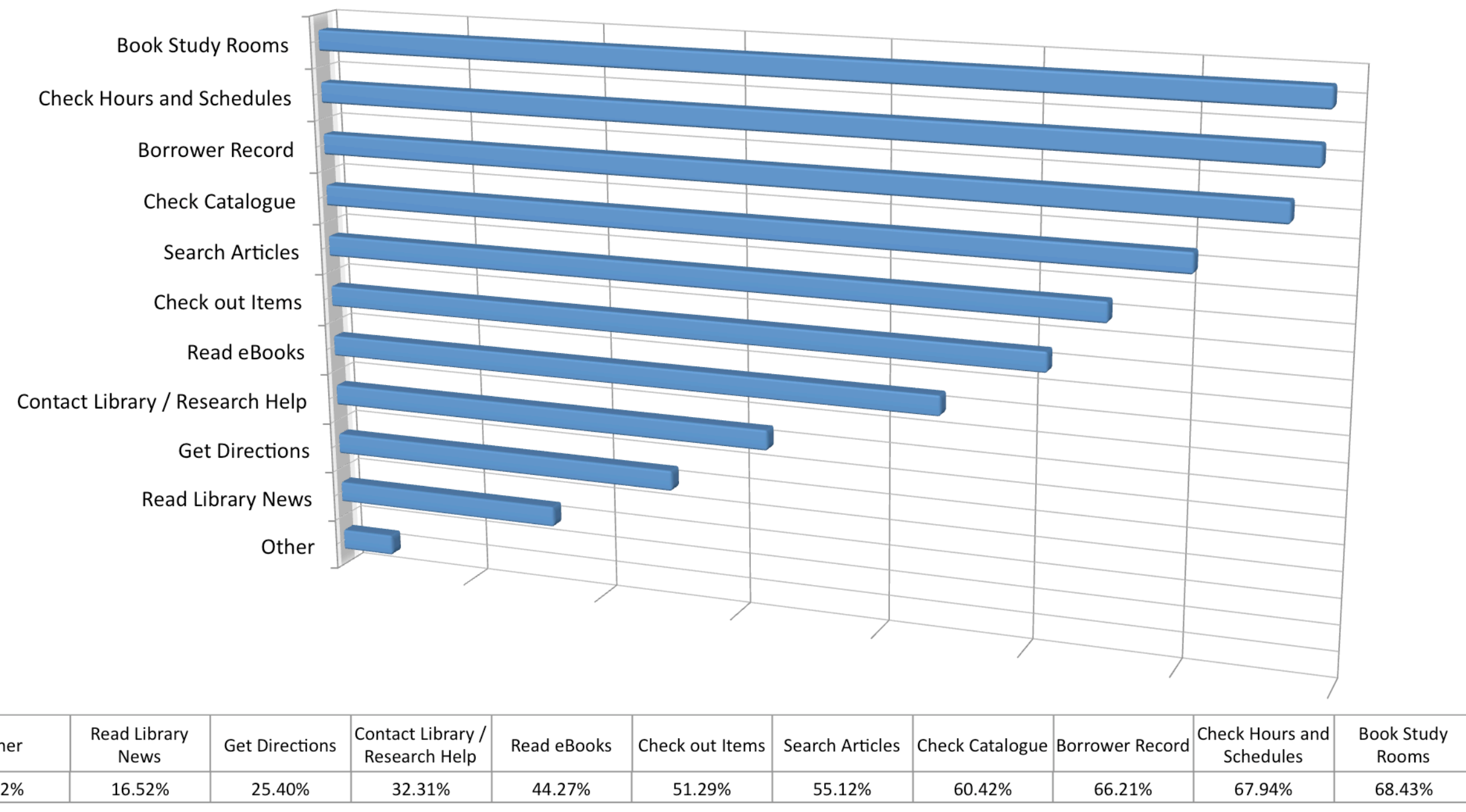




\section{What we learned}

- Approximately $20 \%$ of our population had Internet-enabled smart phones

- Potential for most of our population having these phones within 3 years

- Data plan costs are a deterrent to use

- We should be looking at personalized services 
Library mobile

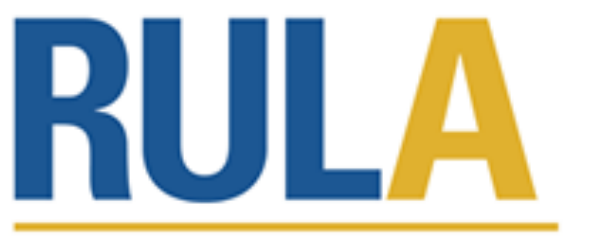

Ryerson University Library \& Archives

\section{Hours}

\section{Book a Room}

Catalogue Search

Check for Available Laptops

Library News

Contact Us

Research Workshops

Full Site | 416-979-5055
- Development

- November 2008 to February 2009

- (ongoing maintenance)

- Staff Involvement

- 2 librarians 


\section{Ryerson Library mobile - Hours}

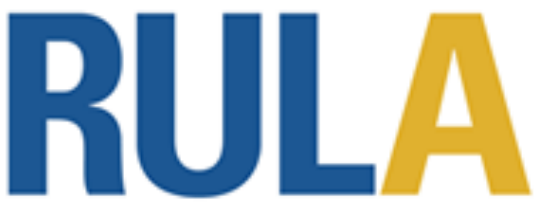

Ryerson University Library \& Archives

\section{Hours}

Book a Room

Catalogue Search

Check for Available Laptops

Library News

Contact Us

Research Workshops

Full Site | 416-979-5055

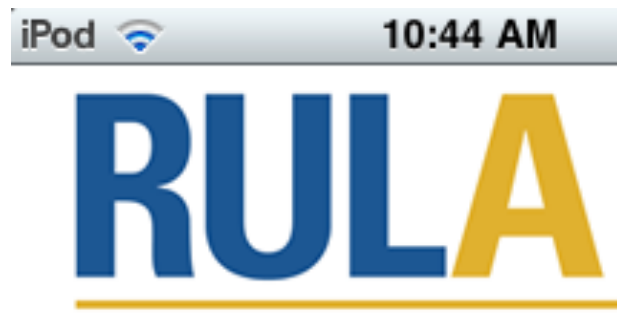

Ryerson University Library \& Archives

\section{Hours}

Today we are open: 10:00AM to Midnight

M. Jan. 4 to Su. Jan. 10, 2010 M \& T: 8:30 am - 7:00 pm

W - F: 8 am - midnight S \& S: 10 am - midnight

M. Jan. 11 - Th Apr 15, 2010 M - F: 8 am - midnight Sa \& Su: 10 am - midnight Closed M. Feb. 15 \& F. Apr. 2, rosn

$\begin{array}{llll}\operatorname{mon} & \sqrt{3}\end{array}$




\section{Ryerson Library mobile - Book a Room}

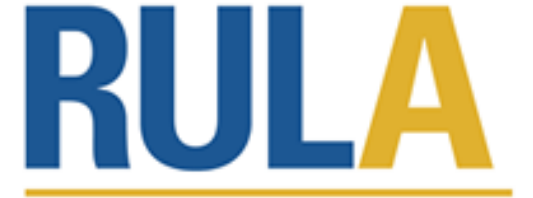

Ryerson University Library \& Archives

\section{Hours}

\section{Book a Room}

Catalogue Search

Check for Available Laptops

\section{Library News}

\section{Contact Us}

\section{Research Workshops}

Full Site | 416-979-5055

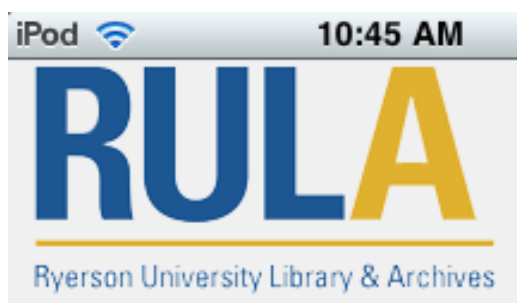

\section{STUDY ROOMS}

Menu:

\begin{tabular}{|l|}
\hline Search Bookings \\
Book A Study Room \\
$\begin{array}{l}\text { View Room Availability } \\
\text { (Within the next 3 hours) }\end{array}$ \\
\hline View Booking Policy \\
\hline \multicolumn{1}{|c|}{} \\
\hline
\end{tabular}

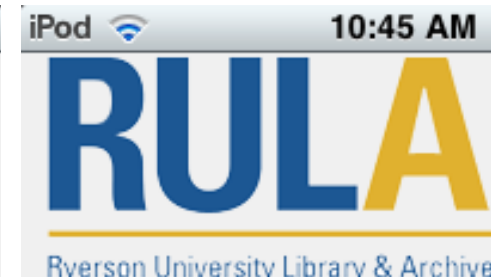

STUDY ROOMS

Book for Today:

11:00AM

LIB856 (4 seats)

Available until 1:00PM

LIB855 (4 seats)

Available until 1:00PM >

\section{LIB759 (8 seats)}

Available until 12:0กPM

त कि $\sqrt{3}$




\section{Ryerson Library mobile - Catalogue}

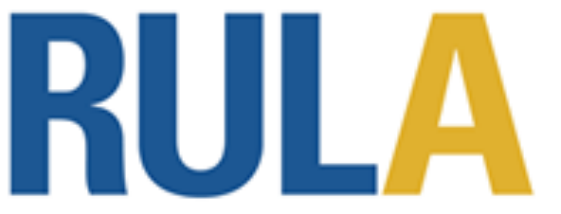

Ryerson University Library \& Archives

\begin{tabular}{|l|}
\hline Hours \\
\hline Book a Room \\
\hline Catalogue Search \\
\hline Check for Available Laptops \\
\hline Library News \\
\hline Contact Us \\
\hline Research Workshops \\
\hline
\end{tabular}

Full Site | 416-979-5055
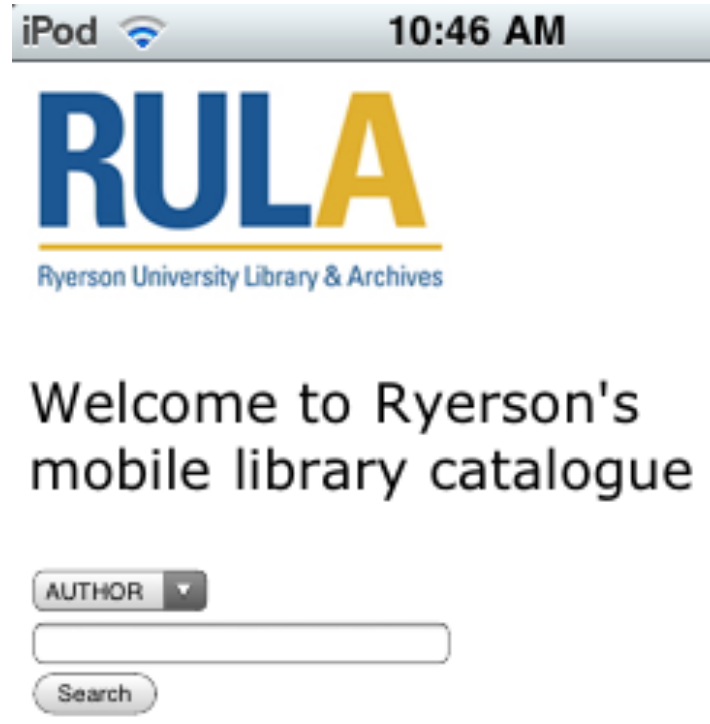

Advanced Searching View your patron information

Need help searching?

$4>+\sqrt{3}$




\section{Ryerson Library mobile - Laptops}

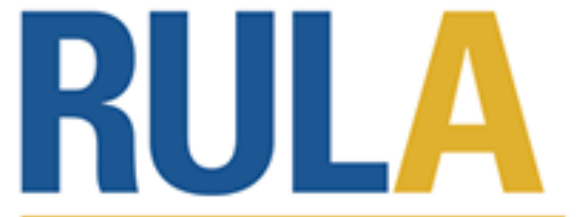

Ryerson University Library \& Archives

\begin{tabular}{|l|}
\hline Hours \\
\hline Book a Room \\
\hline Catalogue Search \\
\hline Check for Available Laptops \\
\hline Library News \\
\hline Contact Us \\
\hline Research Workshops \\
\hline
\end{tabular}

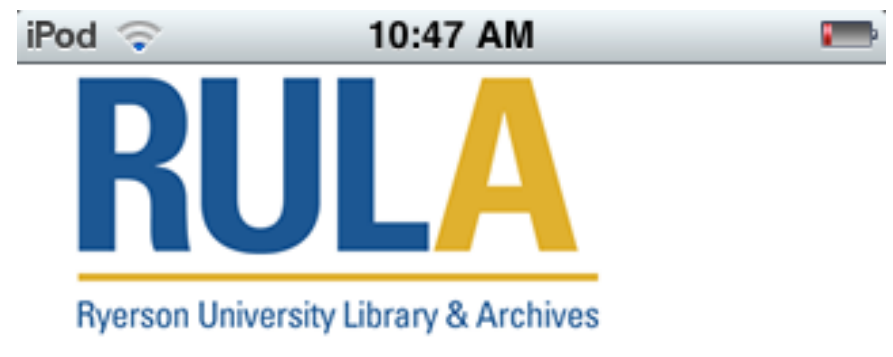

\section{Available Laptops}

There are 64 laptops available in the library.

\section{PC Laptop record \\ MAC Laptop record}

For more information about our Laptops see:

\section{Laptop Loan FAQ}

Full Site | 416-979-5055

$>$ क क $\sqrt{3}$




\section{Ryerson Library mobile - News}

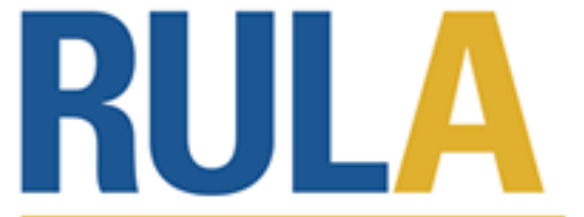

Ryerson University Library \& Archives

\begin{tabular}{|l|}
\hline Hours \\
\hline Book a Room \\
\hline Catalogue Search \\
\hline Check for Available Laptops \\
\hline Library News \\
\hline Contact Us \\
\hline Research Workshops \\
\hline
\end{tabular}

Full Site | 416-979-5055

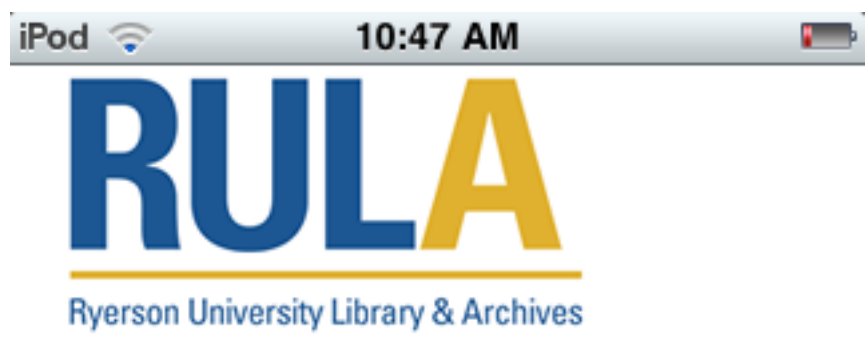

News

Check out our New News Blog

Library Hours During Exams

Quiet Study during Exams

Ask Us...in the Cafeteria!

An Evening with Lenin

4
क त $\sqrt{3}$




\section{Ryerson Library mobile - Research Workshops}

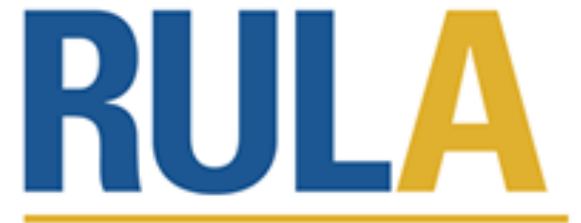

Ryerson University Library \& Archives

\section{Check for Available Laptops}

\section{Library News}

Contact Us

Research Workshops

Full Site | 416-979-5055

\begin{tabular}{|l|}
\hline Ryerson University Library \& Archives \\
\hline Hours \\
\hline Book a Room \\
\hline Catalogue Search \\
\hline Check for Available Laptops \\
\hline Library News \\
\hline Contact Us \\
\hline Research Workshops \\
\hline
\end{tabular}

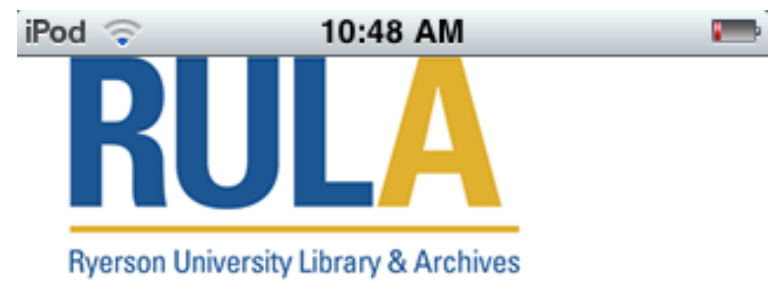

\section{Research Workshops}

All workshops are free but you do need to register.

\section{Mastering the Library's Electronic Resources}

Thurs Jan. 14 5:30 - 6:20 pm LIB393A

Mon Jan. 25 5:30 - 6:20 pm LIB393A

Thurs Feb. 4 2:00 - 3:00 pm LIB393A

Mon Feb. 8 2:00 - 3:00 pm

$<$ - क क

13




\section{Mobile Developments}

Students wanted more than just access to library resources...

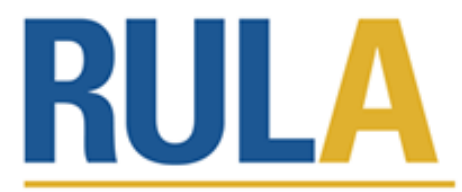

Ryerson University Library \& Archives

\section{Hours}

\section{Book a Room}

\section{Catalogue Search}

\section{Check for Available Laptops}

\section{Library News}

\section{Contact Us}

\section{Research Workshops}

\section{Full Site | 416-979-5055}

Ryerson Library mobile http://m.library.ryerson.ca/

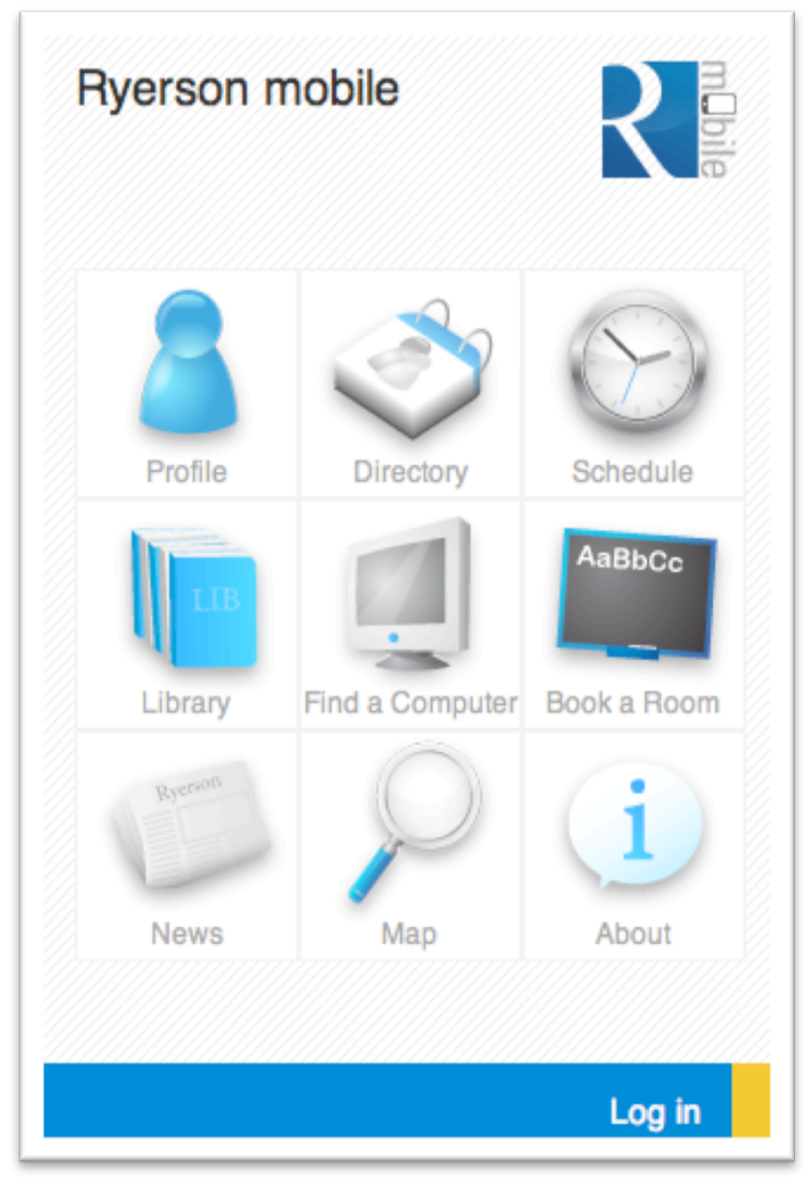

Ryerson mobile http://m.ryerson.ca/ 


\section{Ryerson mobile}

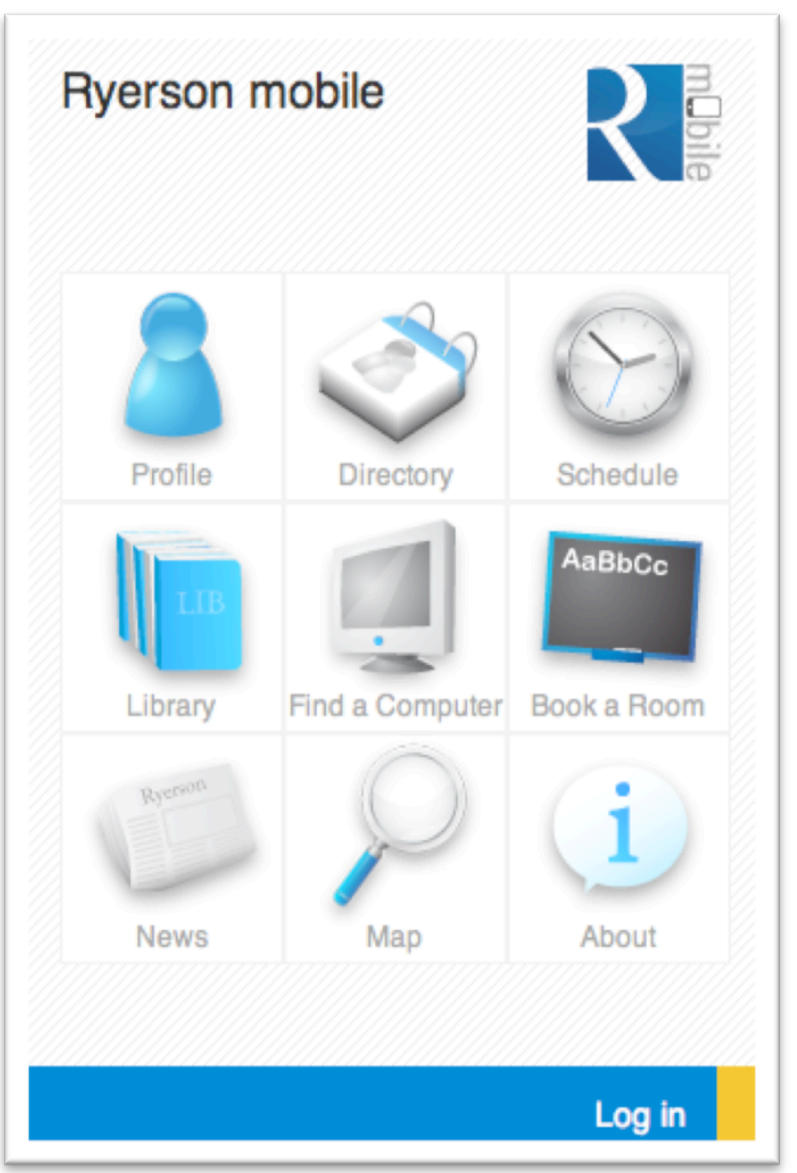

Planning

- February to April 2009

Development - Phase I

- May to August 2009

Launch

- September $14^{\text {th }} 2009$

\section{Staff Involvement}

- Managers:

- 2 librarians

- Development:

- 2 web programmers

- Computer Science and Image Arts students

- 1 graphic designer

- Image Arts student

- Marketing, Student Focus Groups

- SIFE Ryerson (a variety of MBA's and undergrads from the Business School)

- Advisory Roles

- Director of Innovation, Office of the President

- Director of Application Development, CCS (Central Computing) 
Reasons for Library's Involvement in Ryerson mobile

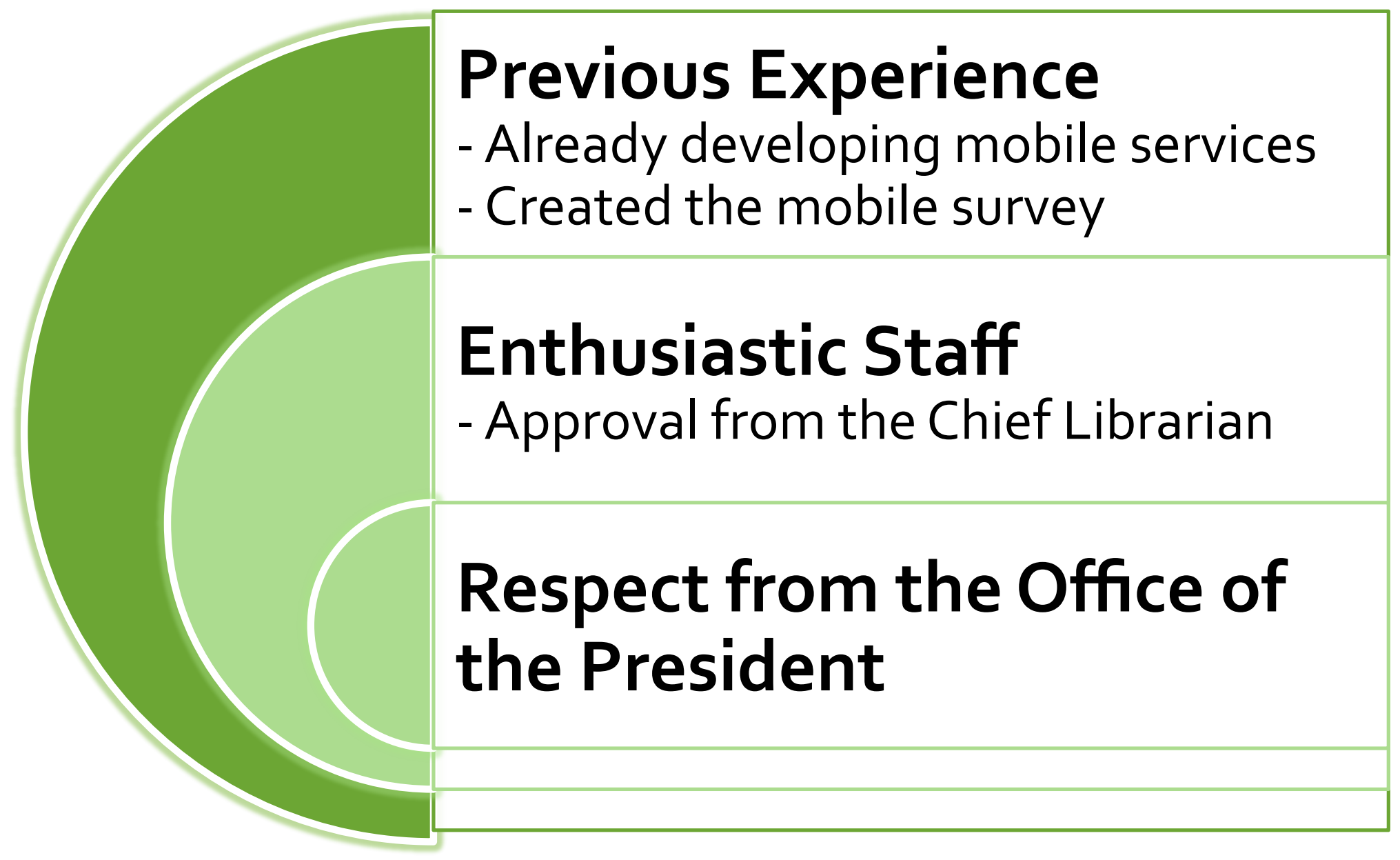




\section{Ryerson mobile - Profile}

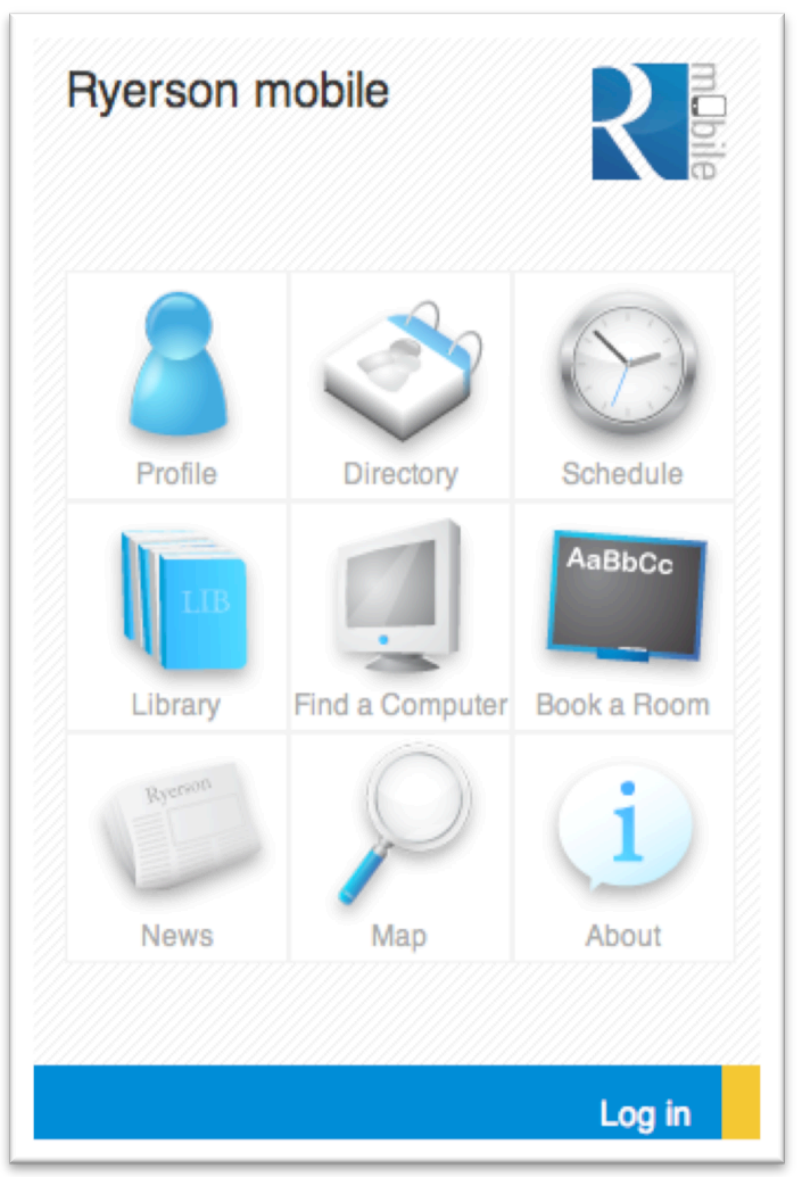

\begin{tabular}{ll} 
iPod $\curvearrowright$ & $12: 00$ \\
\hline Your Profile &
\end{tabular}

News feeds

$\begin{array}{ccc}\text { Title } & \text { Show } \\ \text { RU News } & \text { Articles } \\ \text { Library News } & & \\ \text { RU Research News } & & \\ \text { RU Campus Events } & \end{array}$

Preferences

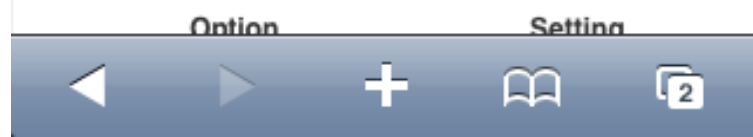




\section{Ryerson mobile - Directory}

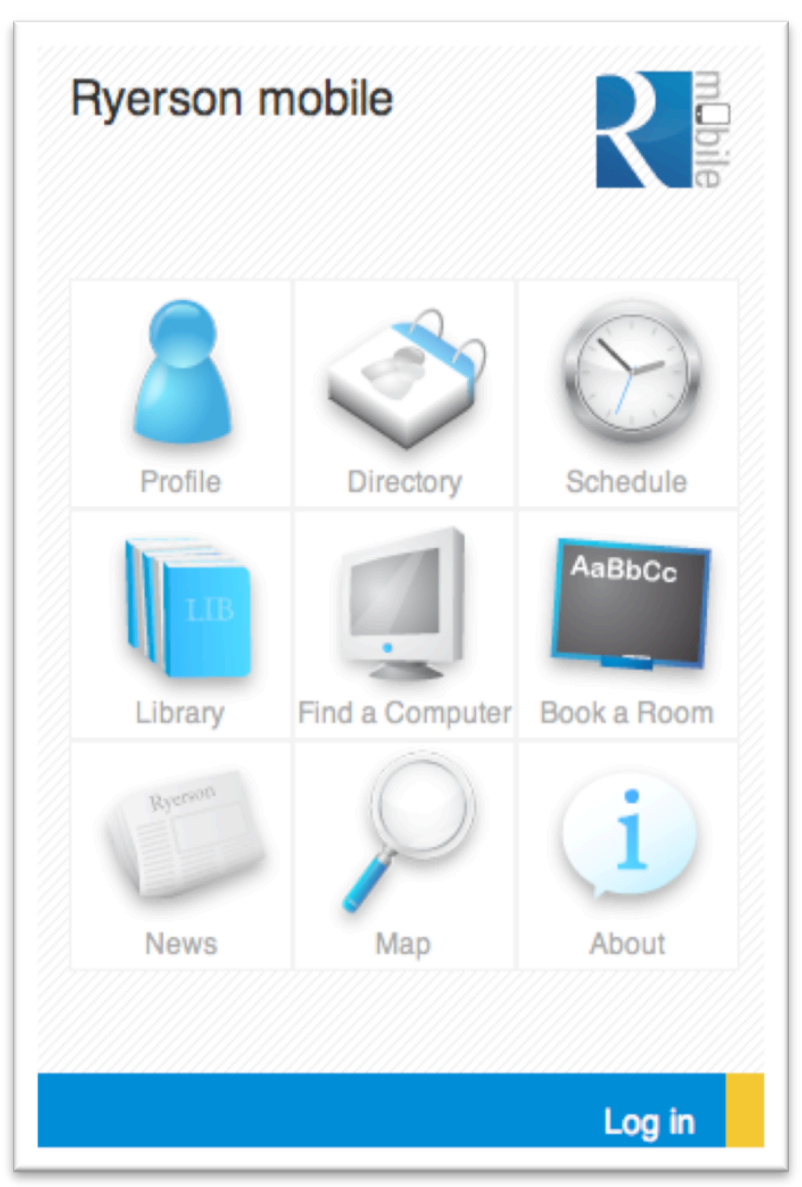

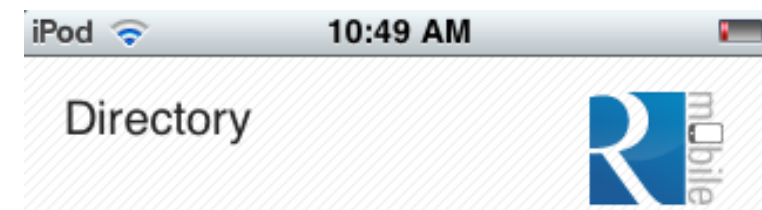

Search By Name
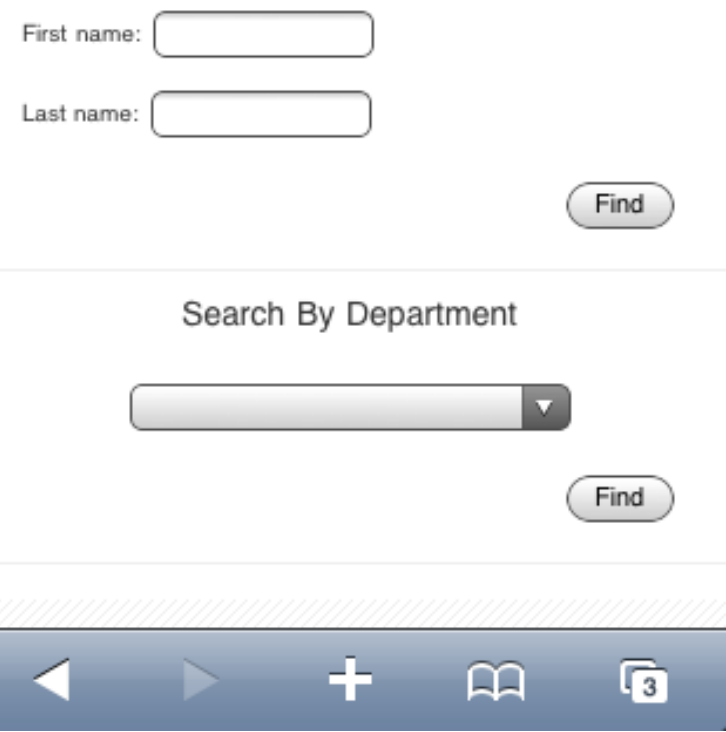

iPod $\approx \quad 10: 49$ AM

Directory

R

\section{Create a new search}

Search result for Graham McCarthy:

Clicking a member's extension will place a call if your phone supports this feature

McCARTHY, Graham

LIBRARY · Programmer I

Location: LIB-667

Extension: 2119

Email: gmccarthy@ryerson.ca

$4>$ क म $\sqrt{3}$




\section{Ryerson mobile - Schedule}

Ryerson mobile
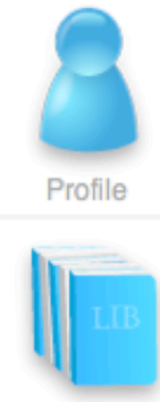

Library

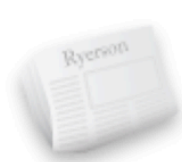

News

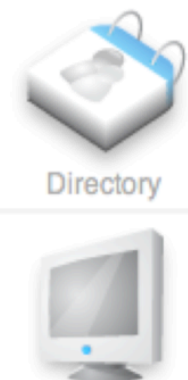

Find a Computer

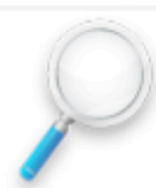

Map
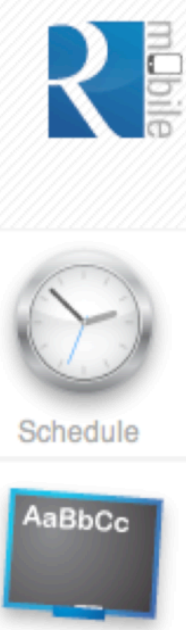

Book a Room

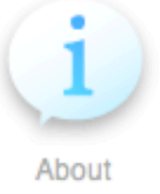

$\log$ in

$\begin{array}{lll}\text { iPod } \curvearrowright & 12: 01 \\ \text { Schedule } & \end{array}$

\section{Browse a new schedule}

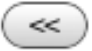

Week Of: Jan 25, 2010
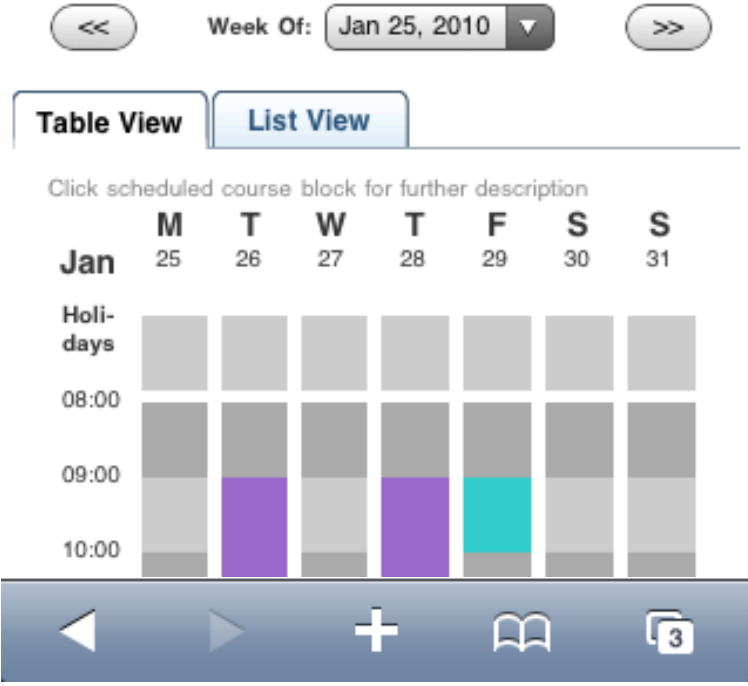

iPod $\approx$

Wednesday, January 06, 2010

BME406 (11:00-12:00)

Biomechanics

Section: EB1

Instructor: McCarter, Thomas

CPS615 (12:00-13:00)

Theory of Parsing

Section: $\mathbf{3 1}$

Room: ENG205

Instructor: Harley, Eric

\section{Thursday, January 07,2010}

ARC720 (12:00-14:00)

Architecture Studio

Section: C01

Room: SHE503

Instructor: Atkinson, Cheryl

\section{Friday, January 08,2010}

$>$ क कि $\sqrt{3}$




\section{Ryerson mobile - Library}

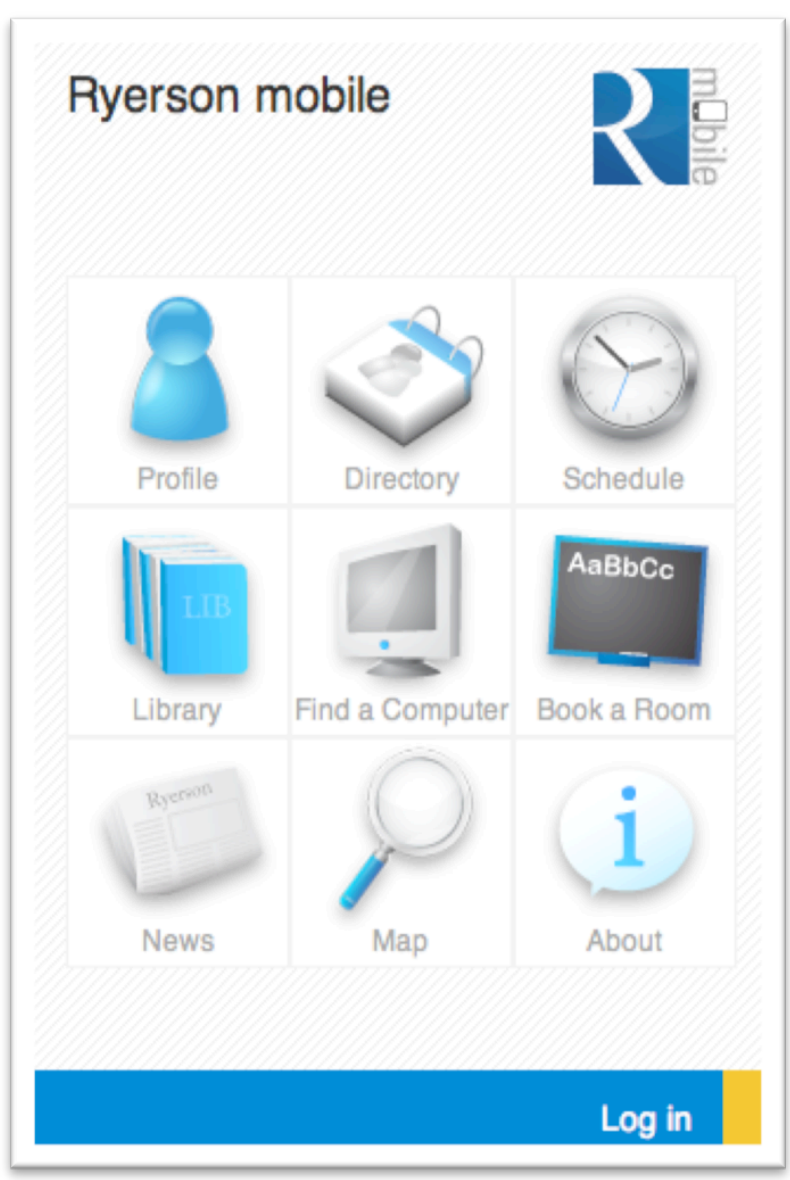

iPod $\% \quad 11: 16$ AM
Library Resources

Search Catalogue >

Manage Library Account >

Audio Tour of the Library (.mp3) >

Contact Information

Library is open from 10:00AM to midnight

Phone : 416-979-5055

Email : refdesk@ryerson.ca

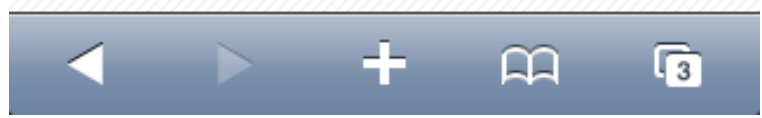




\section{Ryerson mobile - Find a Computer}
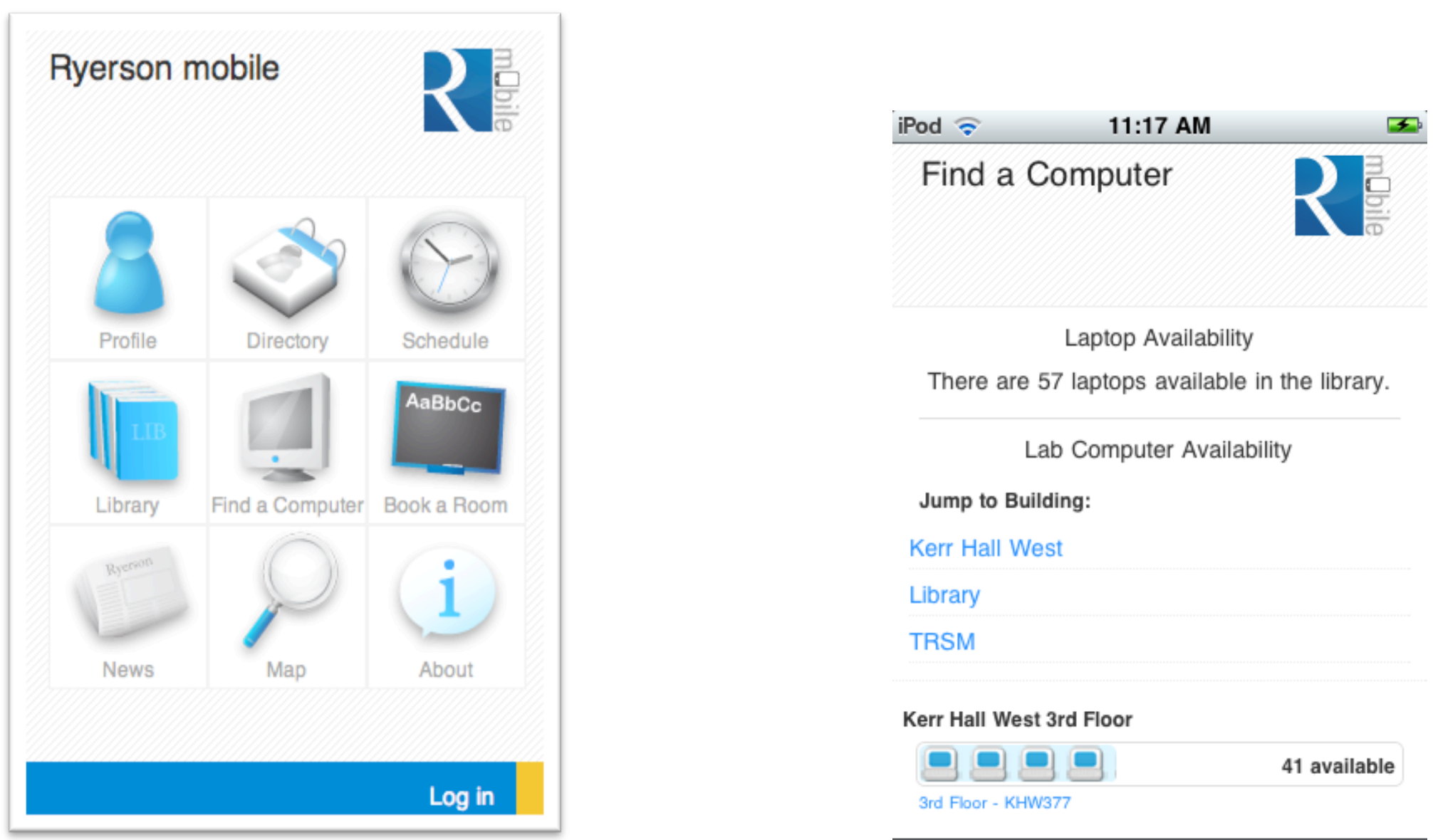

There are 57 laptops available in the library.

Lab Computer Availability

Jump to Building:

Kerr Hall West

Library

TRSM

Kerr Hall West 3rd Floor

$\square \square \square$ 3rd Floor - KHW377 available

$>$ क क




\section{Ryerson mobile-Book a Room}
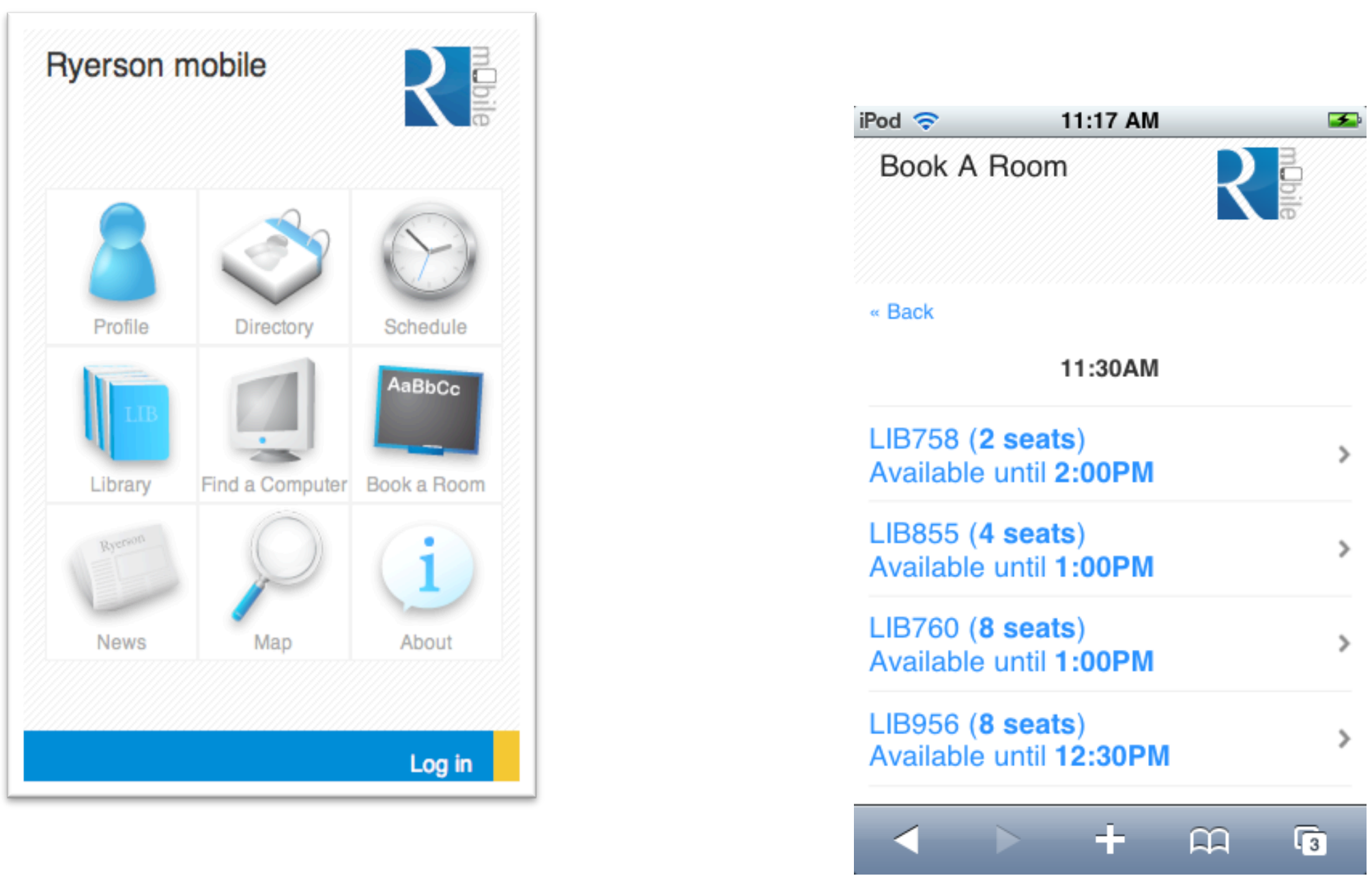


\section{Ryerson mobile-Campus News}

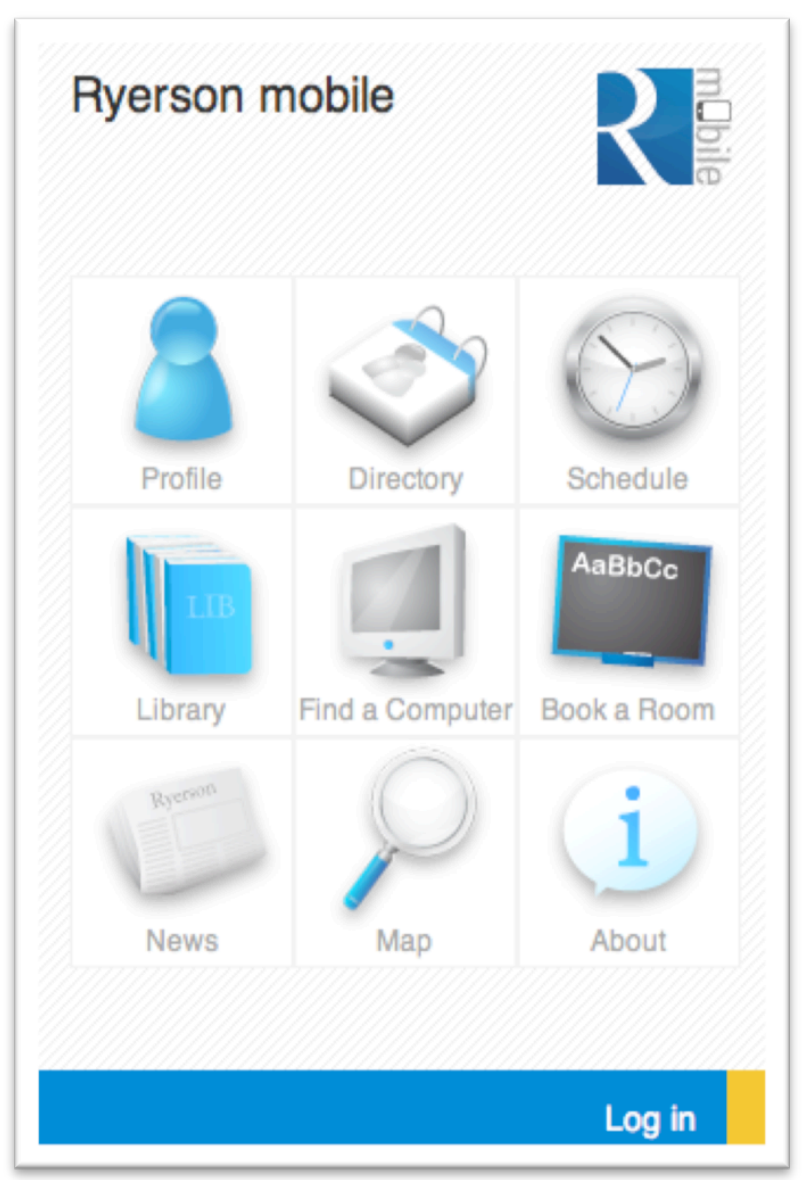
iPod $\% \quad 11: 18$ AM
Ryerson University News
For deaf and hard of hearing gamers, a better experience with the Emoti-Chair Apr 15, 2010

Emoti-Chair enhances gaming experience for deaf, hard of hearing

Aboriginal youth get a taste of food research in university labs

Apr 13, 2010

Researchers host Aboriginal high school students for unique March break internship

Urban planning professor commuting to Harvard University for climate change research role

Apr 09, 2010

In visiting role at Harvard, urban planning

$$
>\quad+\quad \text { कि } \sqrt{3}
$$




\section{Ryerson mobile-Campus Map}
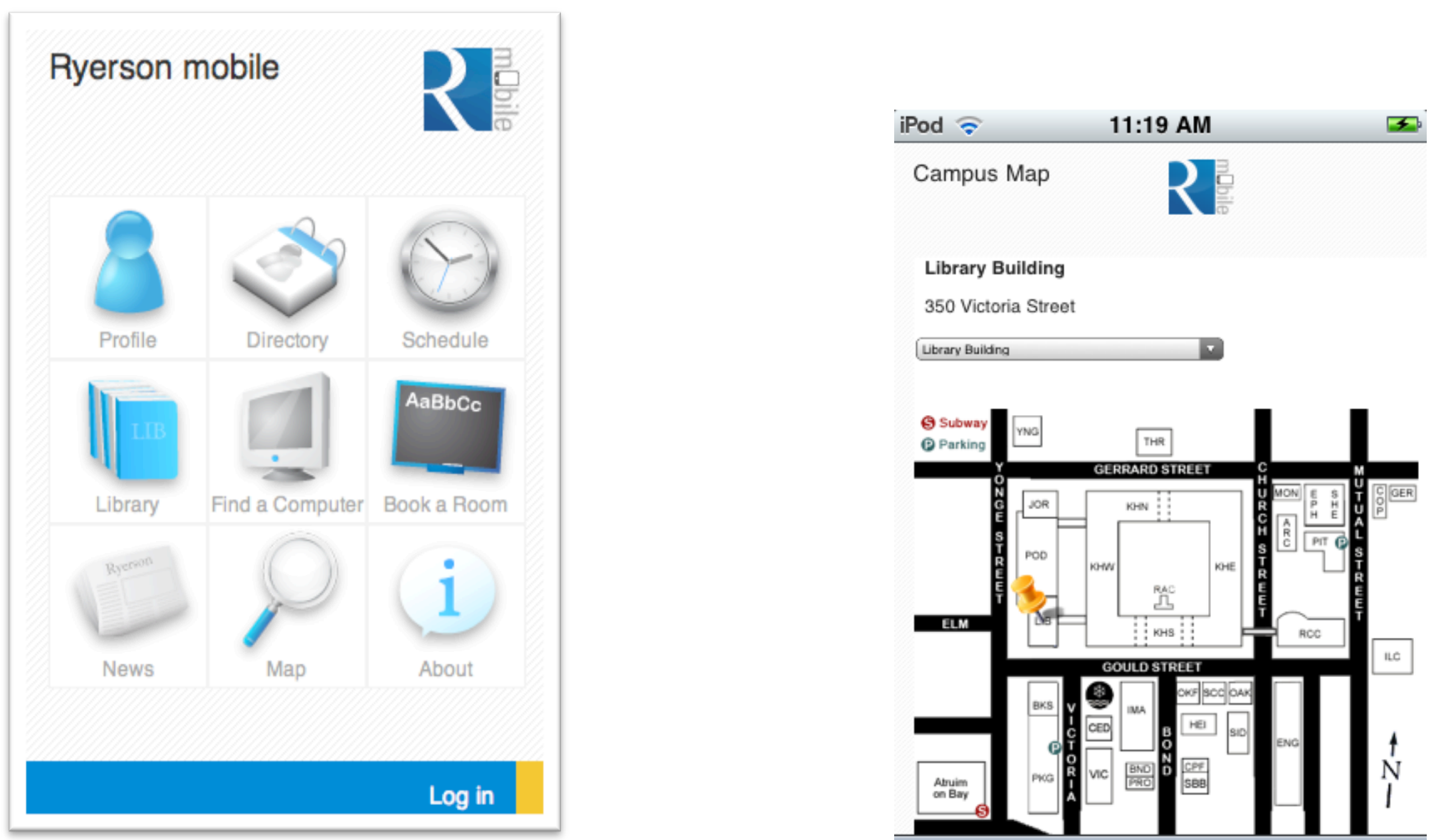

Library Building

350 Victoria Street

ubrary Buildng

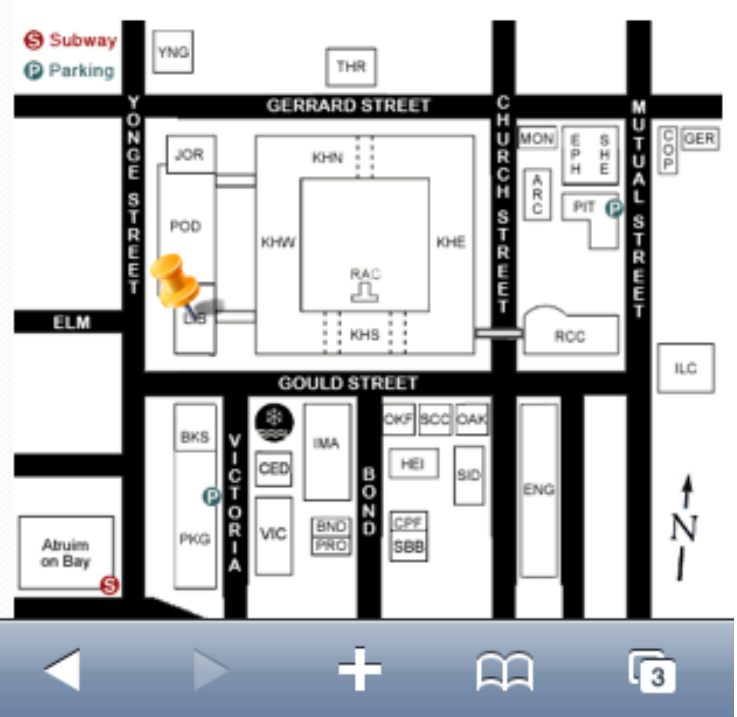




\section{Importance of Involvement}

\section{Benefits}

- Actively participate in a cross campus initiative

- Library can do more than just books

- Strengthening Ties

- Faculties \& Administrative Departments

- Doing what librarians do best

- Enriching student learning experiences

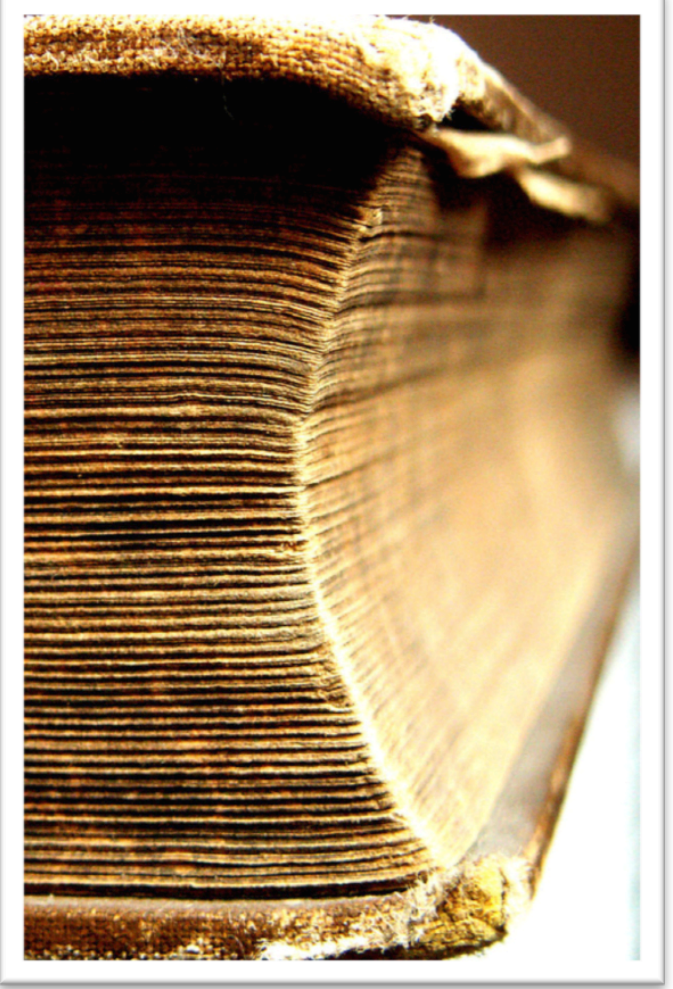




\section{Mobile Survey}

- Ran from Nov. 9-20, 2009

- 760 responses 


\section{Ownership of Internet Capable Mobile Devices}

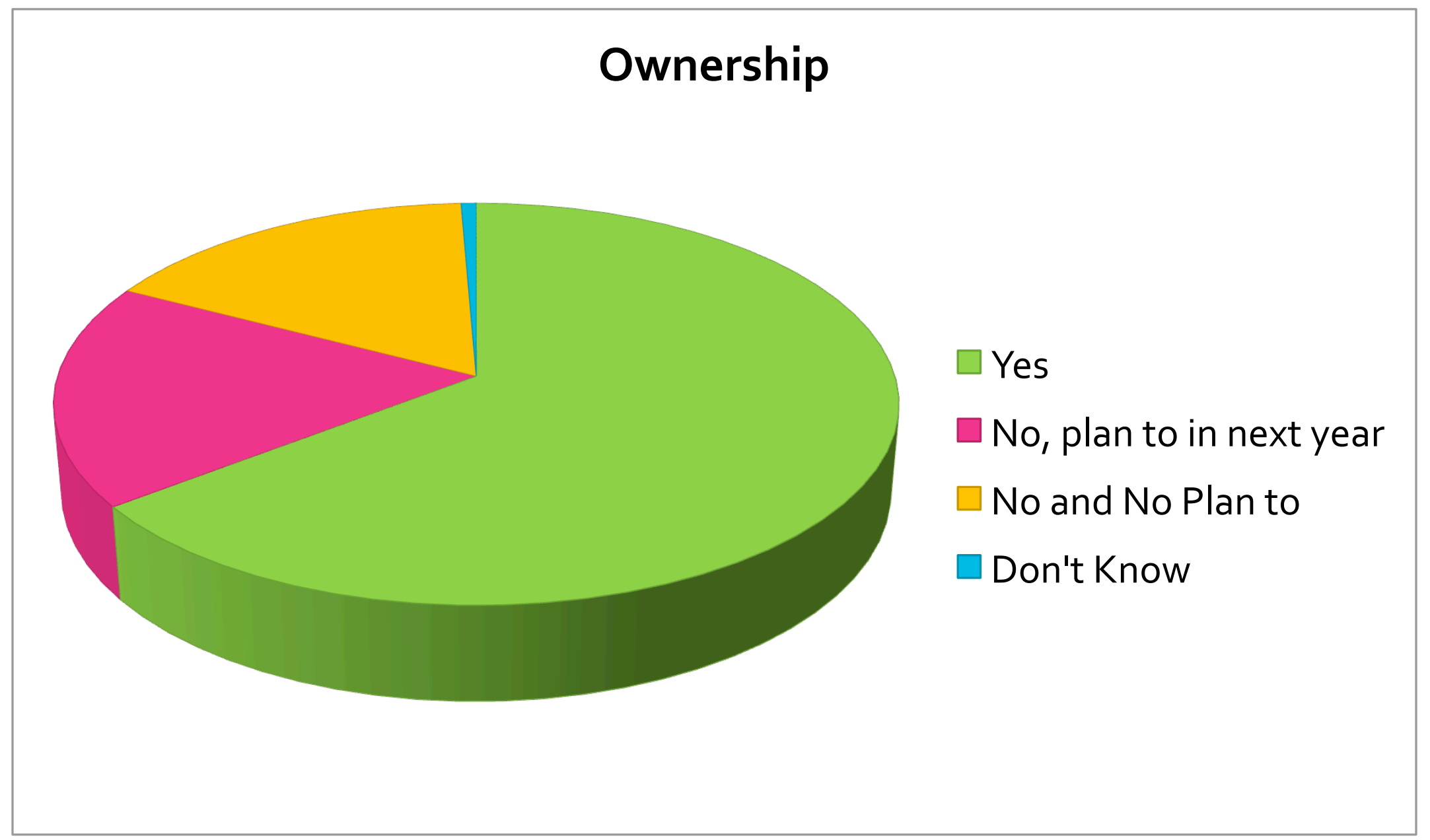




\section{Types of Devices Owned}

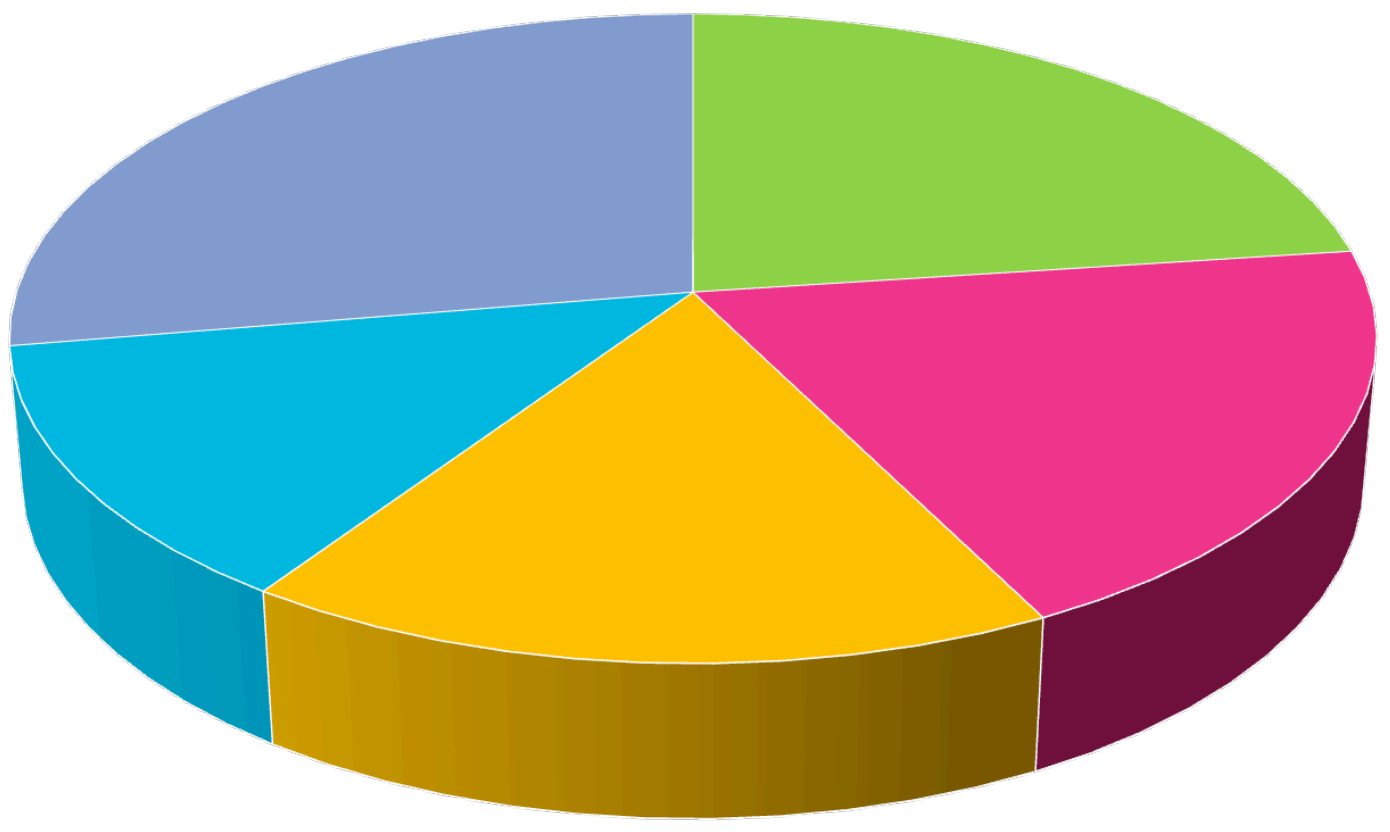

Blackberry

- iPod Touch

-iPhone

- Nokia/Samsung

- Other 


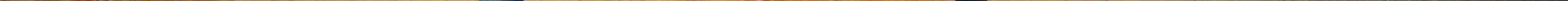




\section{What library services do you use on your mobile device?}

- Book a Room

- Library Hours

- Check Catalogue

- Check Fines/Borrower record

- Check Computer availability 


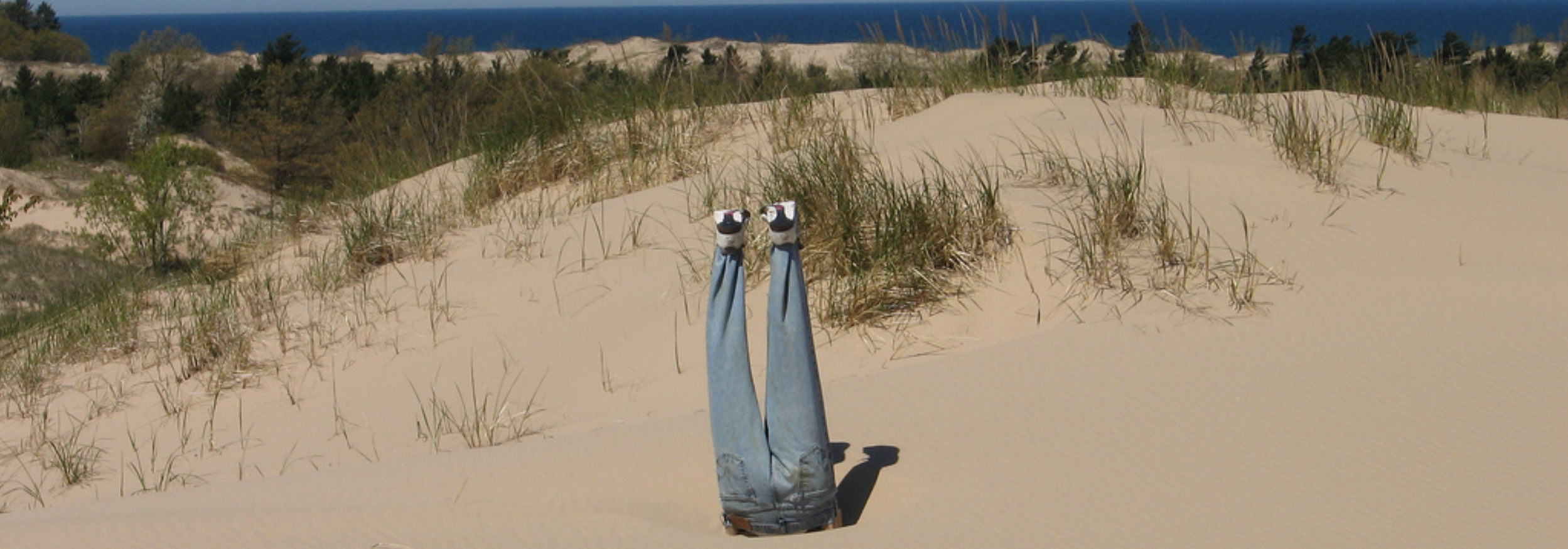

$47 \%$ of respondents with internet-capable mobile devices don't know that Library has mobile services 


\section{Do you own a dedicated ebook reader?}

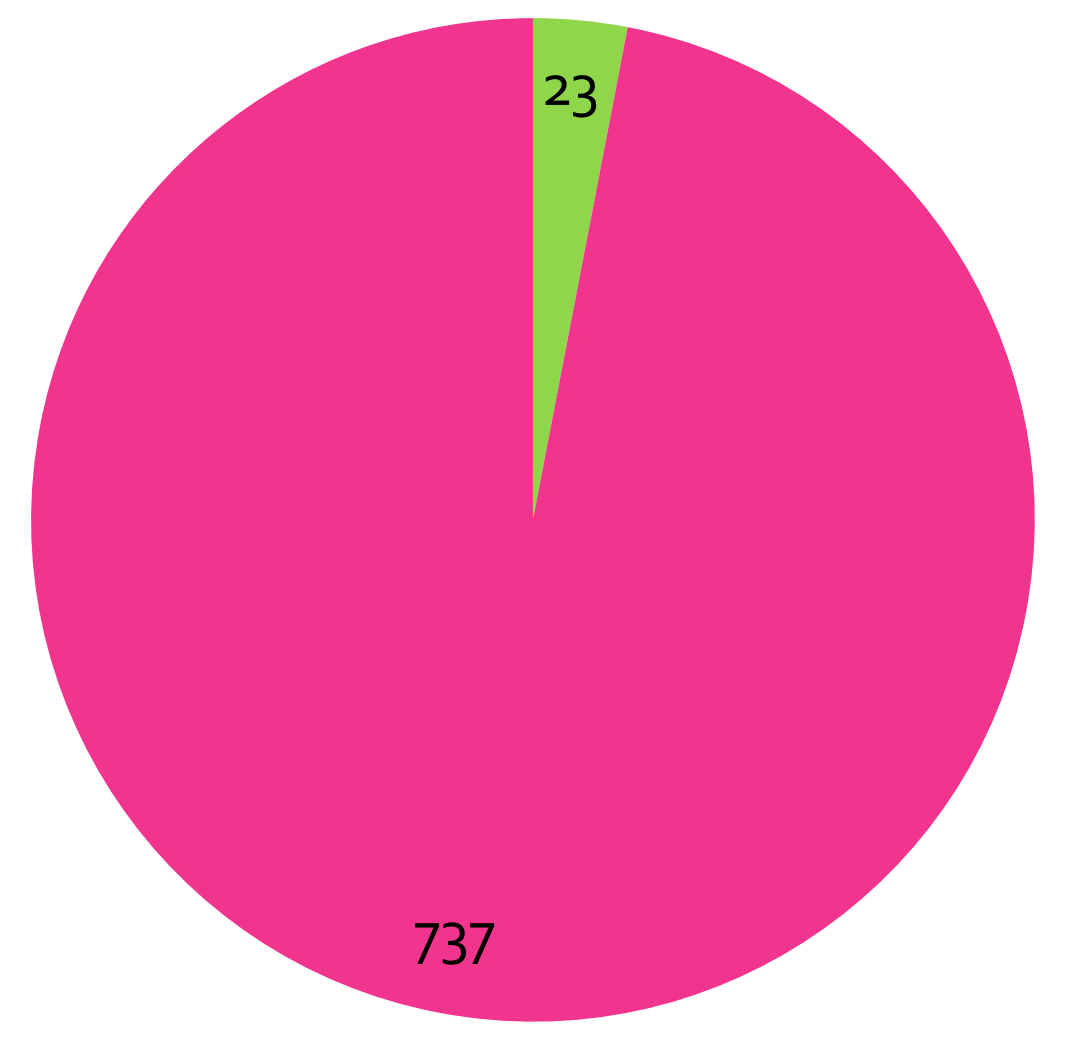

Yes $\square$ No 


\section{New Mobile Library Services \\ Requested}

- Find articles

- Download ebooks

- Check out books

- Contact a librarian for research help 


\section{What we learned from this survey}

- Increase in ownership of internet-capable mobile devices more than we expected

- Device ownership fragmentation

- Free wifi is very important

- Need to do something about ebooks for mobile devices 


\section{Library mobile - June 2010}

- Search for Articles

- News blog

- Iphone catalogue 


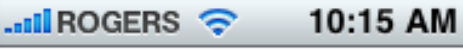

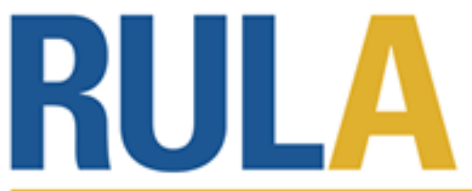

Ryerson University Library \& Archives

\section{Find Articles}

\section{Academic Search Premier}

\section{CINAHL (Nursing)}

\section{Communication and Mass}

Media

\section{Computer Source}

\section{IEEE Xplore (Electrical}

\section{Psychology and Behavioral Science}

For more article resources, visit the Articles and Indexes page on the Library's main website.

Full Site | 416-979-5055

$>$ क की $\sqrt{8}$

\section{Articles}

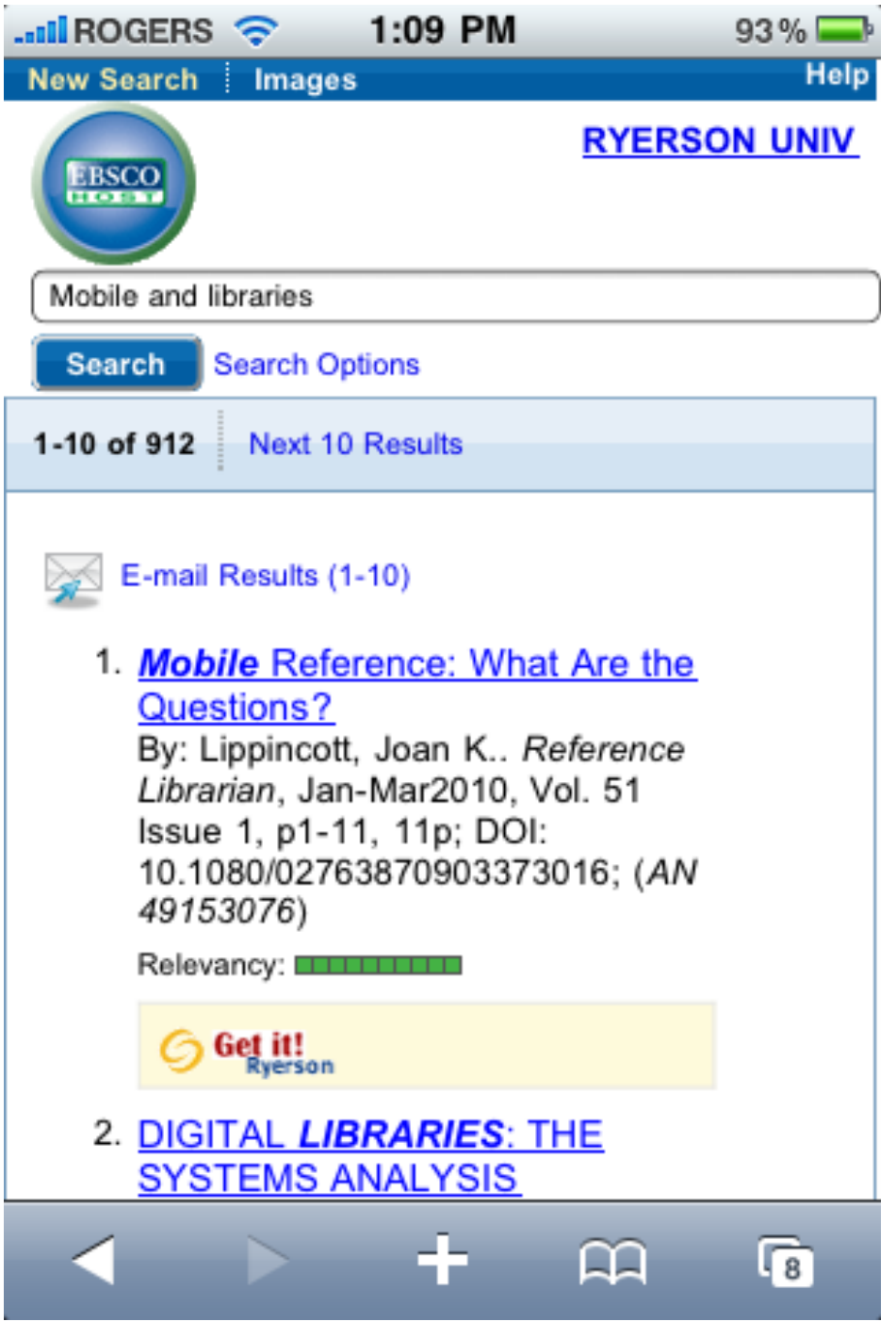




\section{Mobile News Blog}

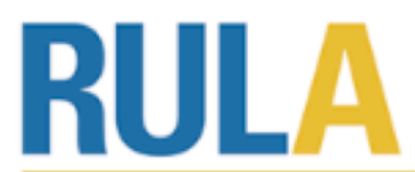

Ryerson University Library \& Archives

Hours

Book a Room

Catalogue Search

Find Articles

Check for Available Laptops

Desktop Computer Availability

Library News

Contact Us

Research Workshops

Ryerson Mobile

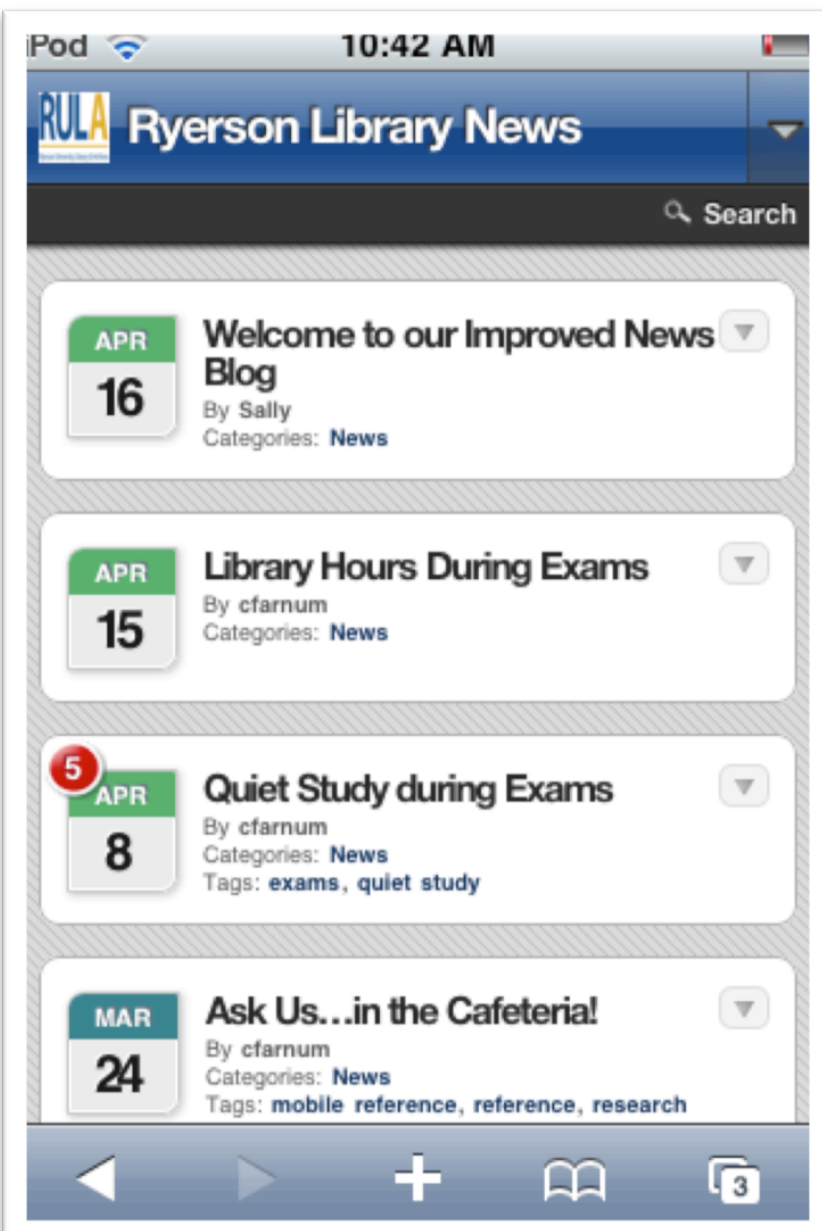




\section{iPhone optimized Catalogue}

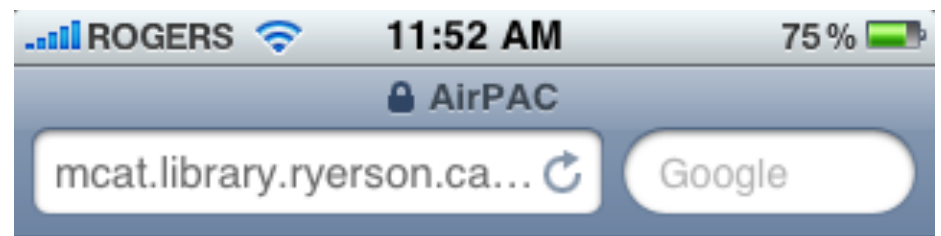

\section{RULA \\ Ayer son Uniwer sity
Library and Mrchimos \\ $M y$
Account}

Search the library catalog:

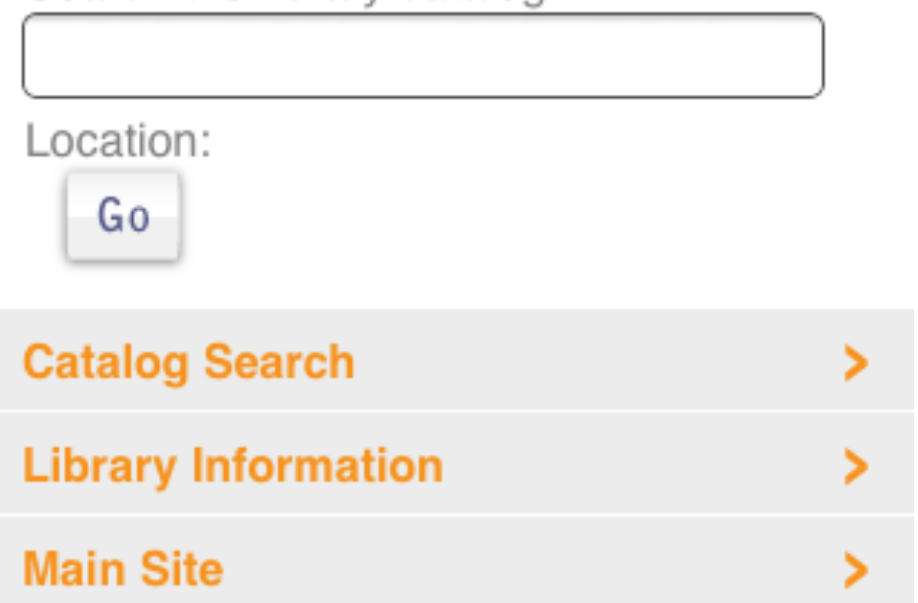

AirPAC (c) Innovative Interfaces, Inc.

$$
\therefore \text { की } \sqrt{8}
$$

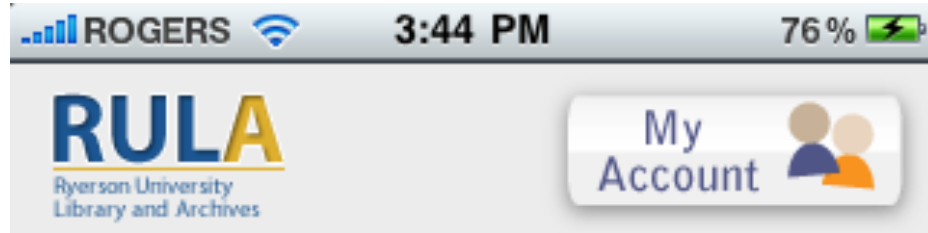

Web design library Go

66 found

$1 \underline{2} \underline{3} \underline{4} \underline{5} \underline{6} \underline{\mathrm{Next}}$

1. XHTML and CSS essentials for library Web design

Sauers, Michael P.

c2006

1 copy available at 10 th Floor

(1) 2. Networking: a beginner's guide

Hallberg, Bruce A., 1964-

5th ed. c2010

1 convavailable at Bverson

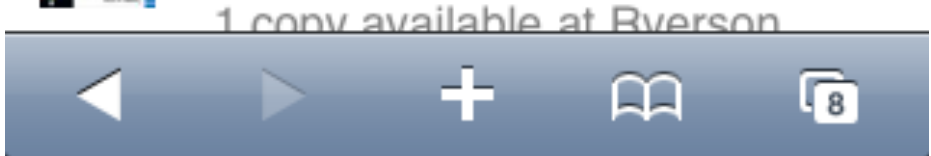




\section{What are our statistics telling us?}

- PercentMobile

- Google Analytics

- WebTrends

- Home-grown systems 

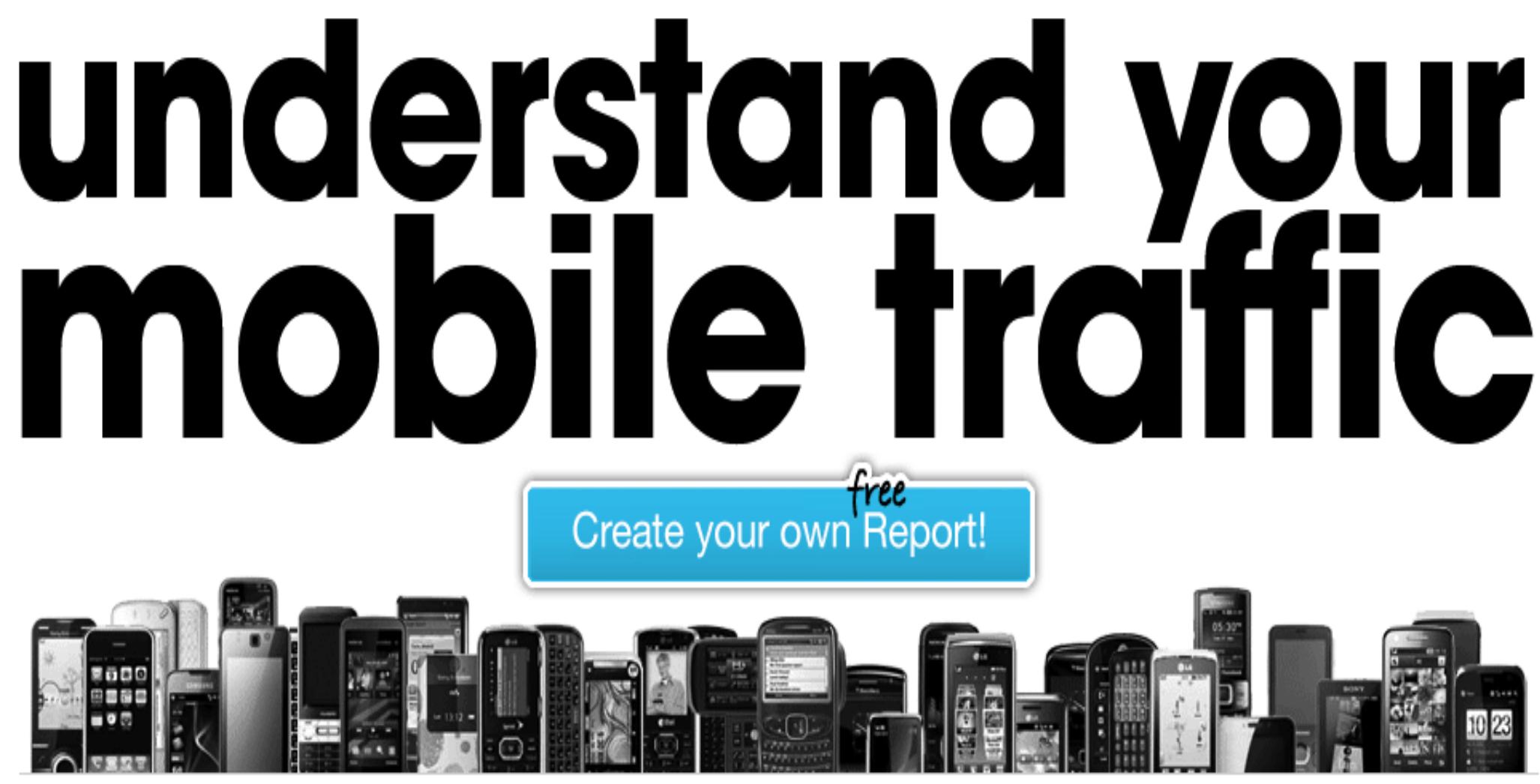

PercentMobile www.percentmobile.com 


\section{PercentMobile}

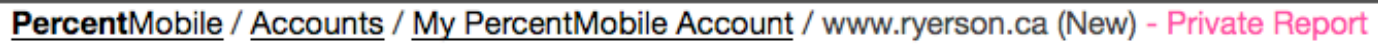

swilson@ryerson.ca Sign Out

\begin{tabular}{|c|c|}
\hline$\%$ & Filters \\
\hline PercentMobile & Hint: Create a filter by clicking a linked item. \\
\hline
\end{tabular}

Visits

\section{Visits by Day}

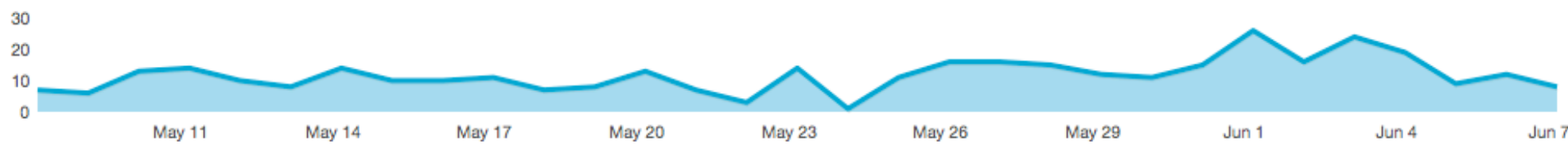

We spotted some iPad activity on your site. Very cool! $\mathbf{E}$ tweet this

\begin{tabular}{|c|c|c|c|c|c|c|c|}
\hline Devices & WiFi Capable & Touchscreen & Full Keyboard & Feature-Phones & Smart-Phones & Experience-Phones & Non-Phones \\
\hline
\end{tabular}

\section{Devices by Visits}

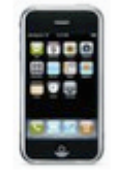

$37.2 \%$

Apple

iPhone OS

$\underline{3 . x}$

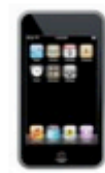

$18.6 \%$

Apple

Pod Touch

OS 3.x

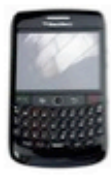

$6.0 \%$

BlackBerny

$\underline{9700}$

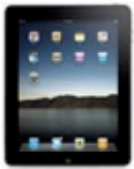

$5.7 \%$

Apple

Pad

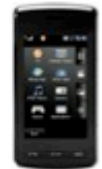

$4.4 \%$

LG

$\underline{\mathrm{LX}} 9700$

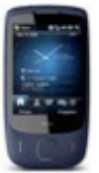

$3.8 \%$

Touch $3 \mathrm{G}$

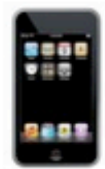

$2.7 \%$

Apple

Pod Touch

os 2.x 


\section{Google Analytics}

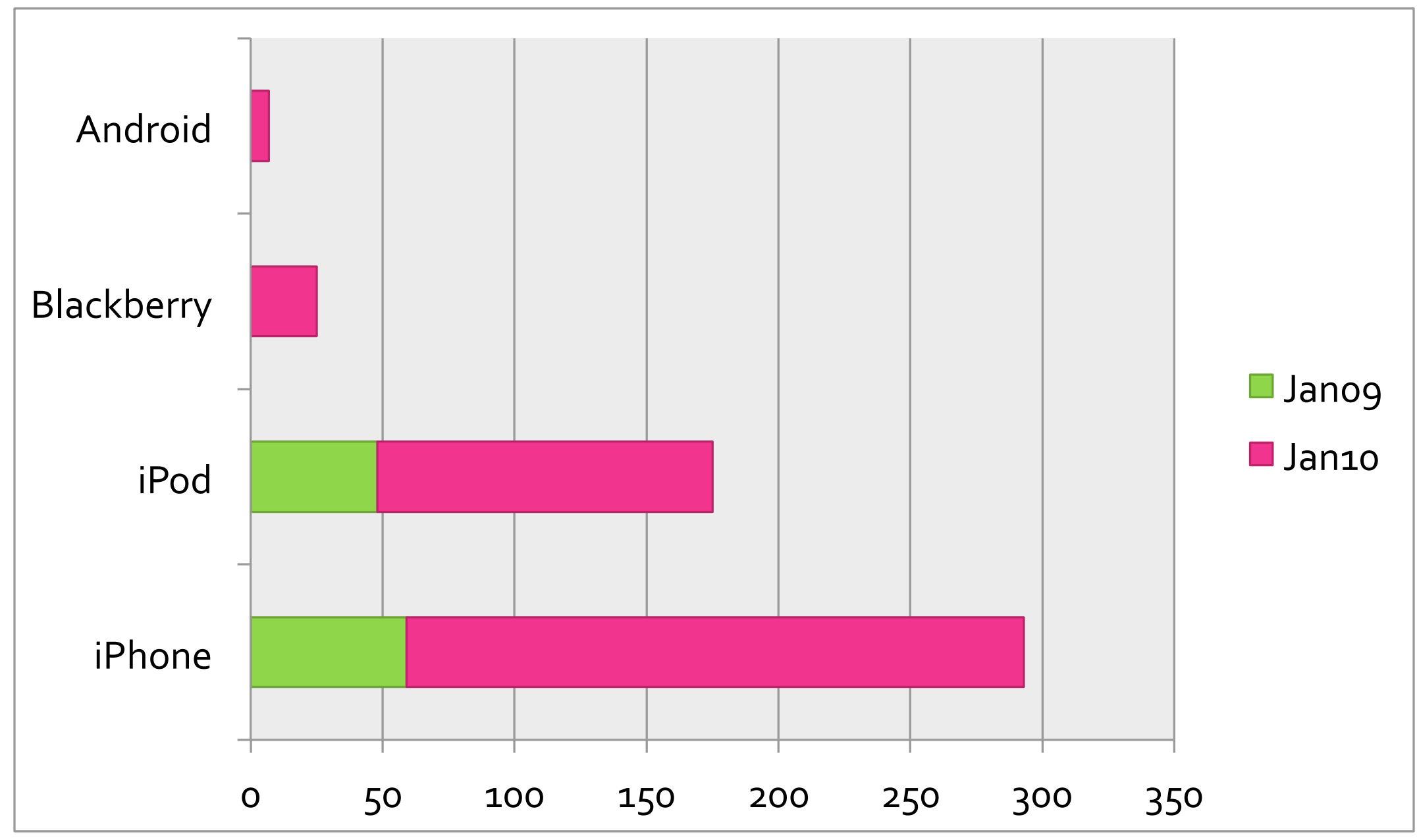




\section{Google Analytics - Devices}

\begin{tabular}{|l|l|r|r|}
\hline & Operating System $\approx$ & Visits $\downarrow$ & Pages $/$ isit \\
\hline 1. & iPhone & 228 & 3.27 \\
\hline 2. & iPod & 98 & 3.49 \\
\hline 3. & Android & 18 & 2.11 \\
\hline 4. & BlackBerry & 11 & 2.82 \\
\hline 5. & SymbianOS & 4 & 3.25 \\
\hline 6. & Windows & 2 & 2.00 \\
\hline 7. & Nokia & 1 & 6.00 \\
\hline 8. & iPad & 1 & 2.00 \\
\hline
\end{tabular}




\section{WebTrends}

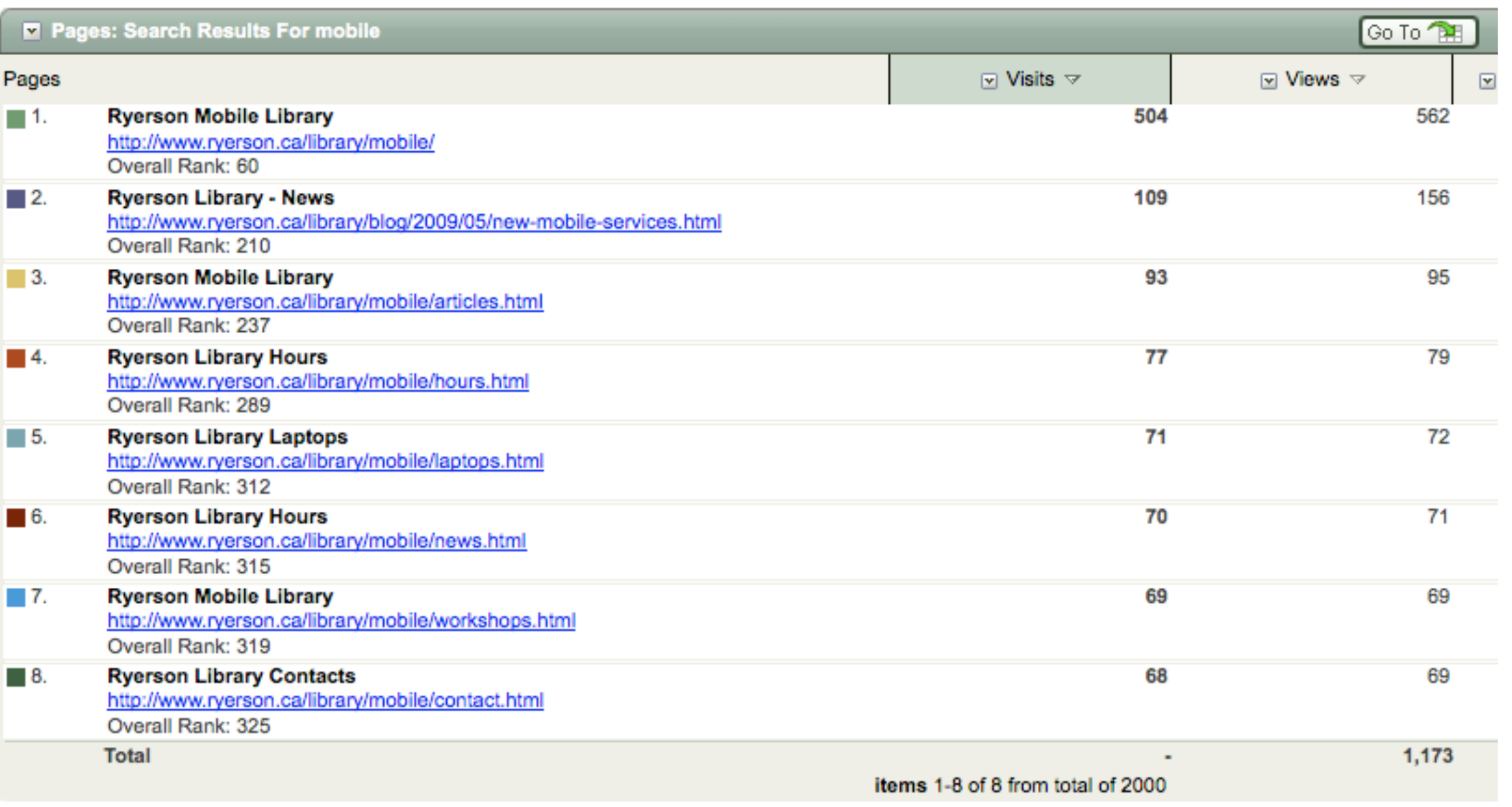




\section{Ryerson Mobile Usage}

(Sept. 14, 2009-Apr. 23, 2010)

\section{Times Used}

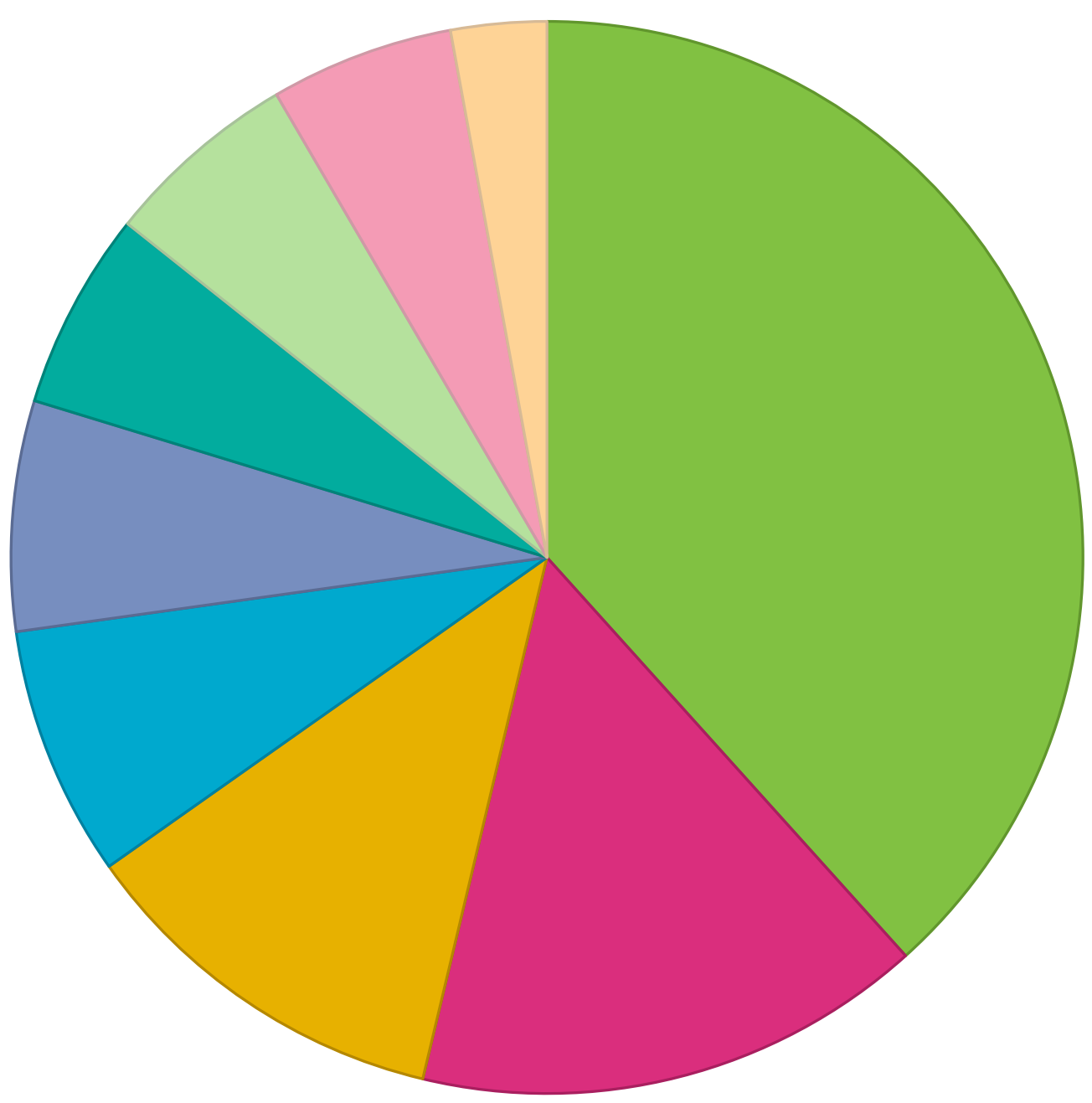

$(3,276$ Unique Users)

$\square$ Schedule

$\square$ Profile

$\square$ Book a Room

$\square$ Directory

$\square$ Computers

$\square$ Map

$\square$ Library

$\square$ News

$\square$ About 


\section{Home Grown Statistics}

\section{Texting from Catalogue - March 2010}

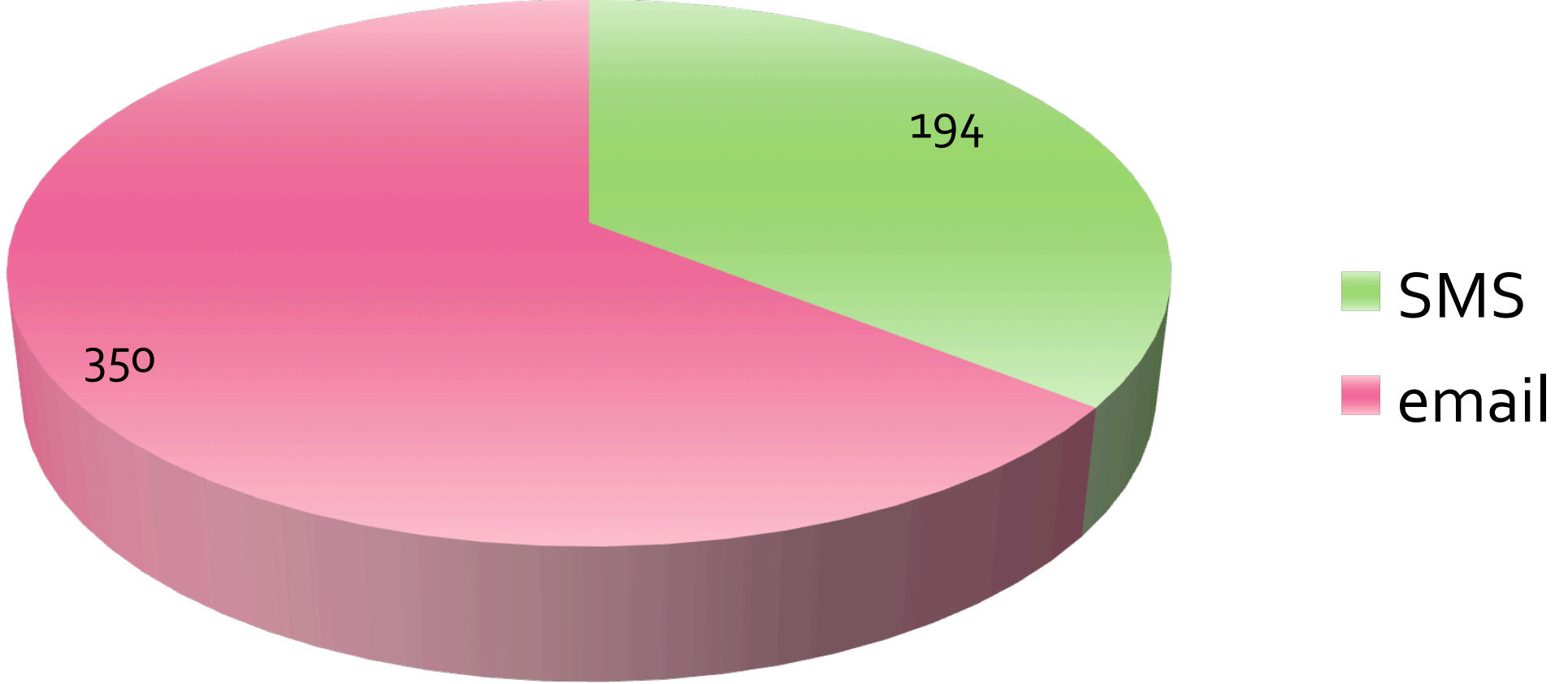




\section{Library mobile}

Upcoming Projects

\section{Summer/Fall 2010 Projects}

\section{Prettier Interfaces}

Mobile Research Guides

Way Finding in the Library (with Indoor Positioning)

\section{Examples:}

-Find books in the stacks

- Find a librarians office

-Find a photocopier

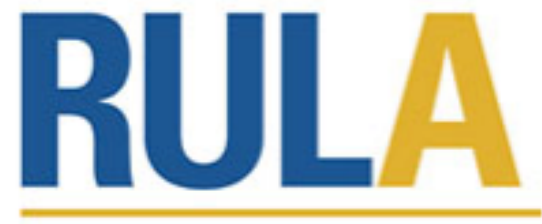

Ryerson University Library \& Archives

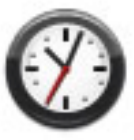

Check hours Book a room

Search the catalogue

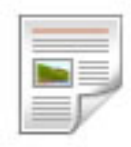

Find Articles

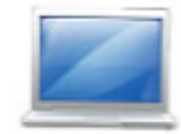

Check for available

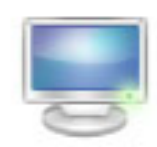

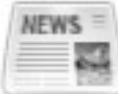
laptops

Desktop computer availability

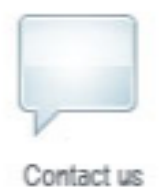




\section{Ryerson mobile}

Upcoming Projects

Summer 2010 Projects

Blackboard Announcements Campus Way Finding Notification System Ryerson Soap Box Varsity Sports Schedules
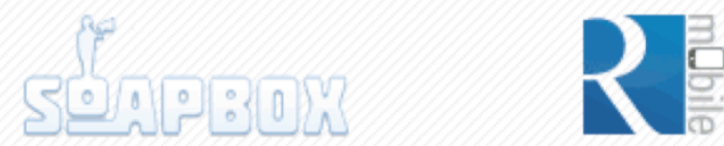

I want less email going to my RyeMail. Put all the announcements into one email and send only one email a day per program. This makes it harder for important emails to get ignored.

Brennan, TRSM April 2nd 2010 4:21pm

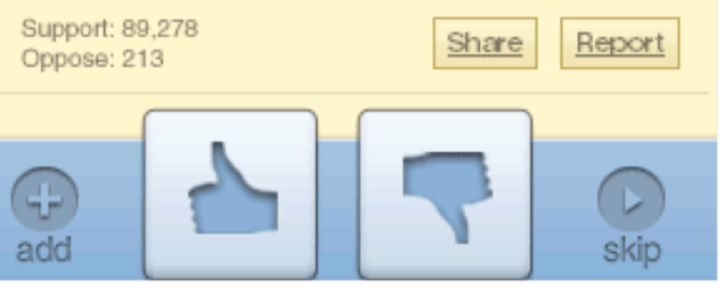

(Manually Set Starting Point) I Auto-Update: (Off †I Go to: (LIB \$) or (Go to my next class)

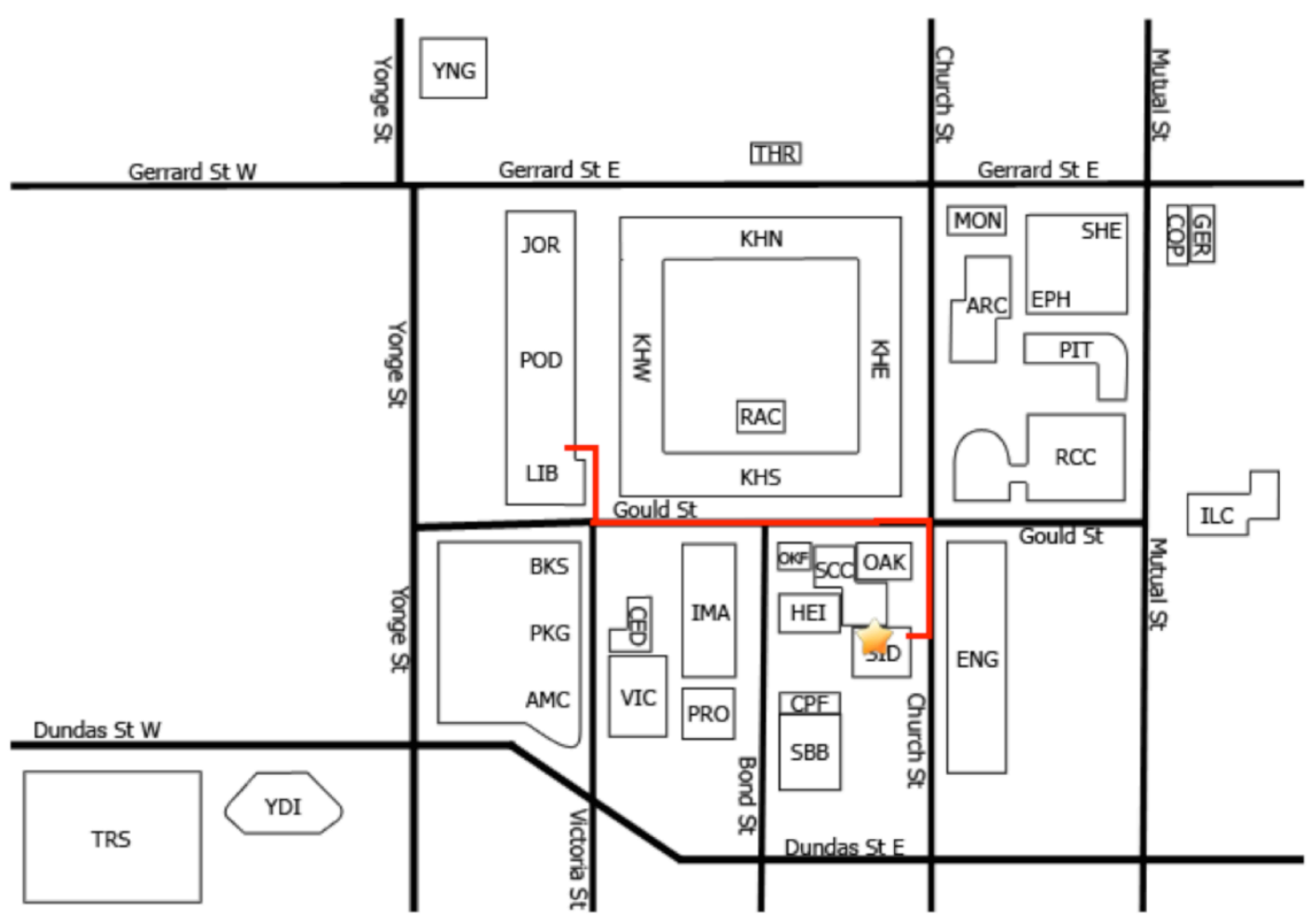

Click on a building to view interior map.

Your browser must support geolocation to determine your location automatically. If your browser lacks support or your location is inaccurate, you may manually set your current location. 


\section{DevelopmentTips:}

Things to keep in mind before creating mobile services

\section{Fast. Obvious. Easy.}

FAST. OBHDUS. EASY.
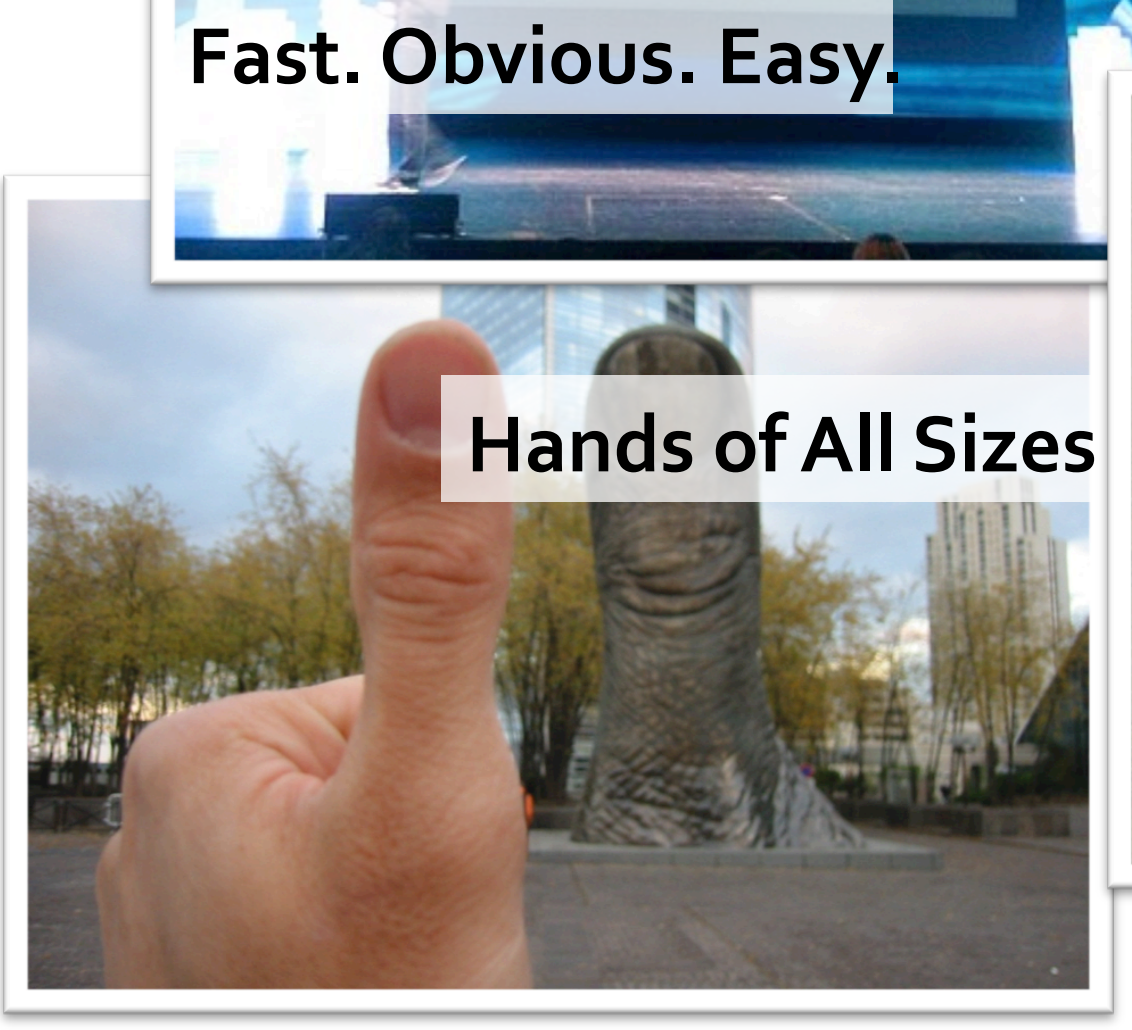

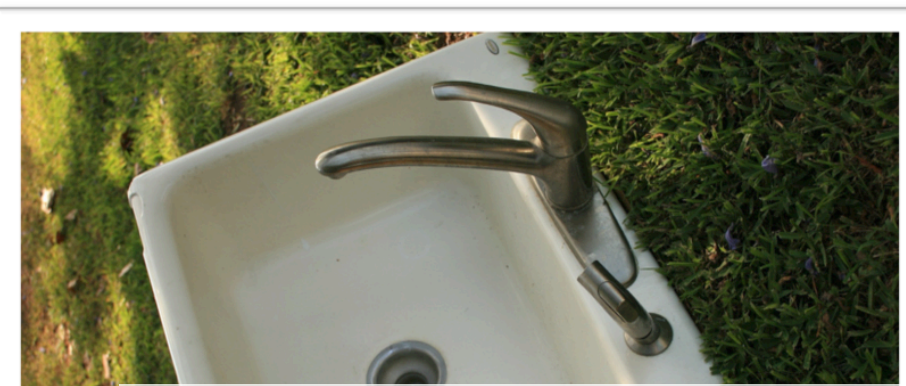

Relevant Information
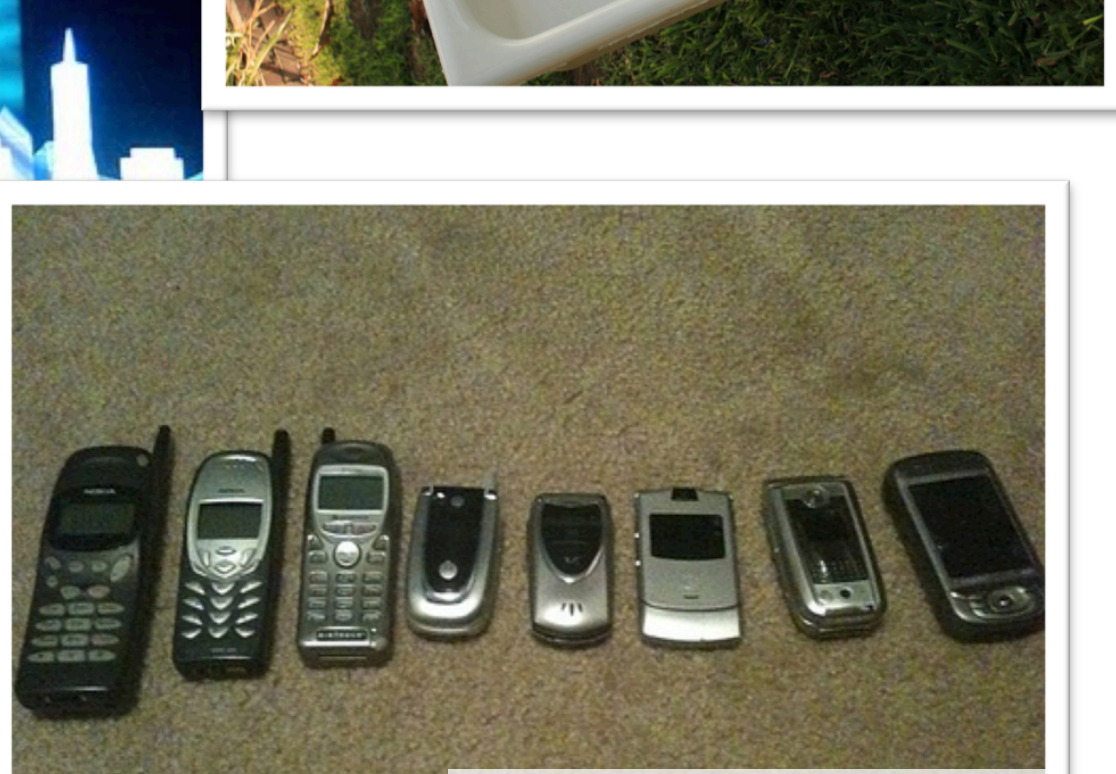

Don't forget Us! 


\section{Thank you}

\section{Survey Results}

2008 results - http://www.ryerson.ca/library/msurvey/

2009 results - http://www.ryerson.ca/library/msurvey2009/

\section{Paper}

"The Mobile University: From the Library to the Campus"

Reference Services Review Volume: 38 Issue: 2

ISSN: 0090-7324

\section{Links}

Ryerson Library Mobile - http://m. library.ryerson.ca

Ryerson mobile - http://m.ryerson.ca

\section{Contact:}

Graham McCarthy - gmccarthy@ryerson.ca

Sally Wilson - swilson@ryerson.ca 